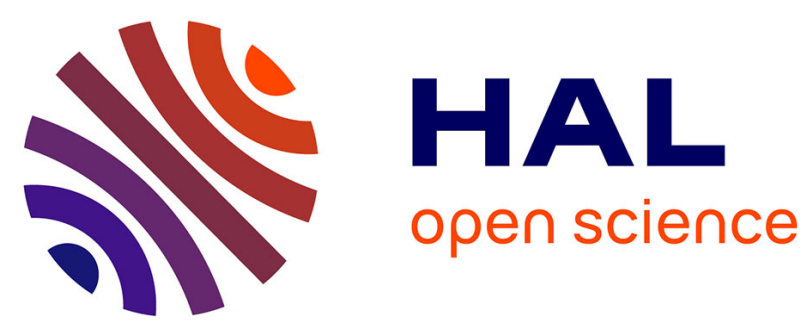

\title{
Magnetic impurities in nanotubes: From density functional theory to Kondo many-body effects
}

P. P. Baruselli, R Fabrizio, Alexander Smogunov, R Requist, E P Tosatti

\section{To cite this version:}

P. P. Baruselli, R Fabrizio, Alexander Smogunov, R Requist, E P Tosatti. Magnetic impurities in nanotubes: From density functional theory to Kondo many-body effects. Physical Review B: Condensed Matter and Materials Physics (1998-2015), 2013, 88, pp.245426. 10.1103/PhysRevB.88.245426 . cea01481347

\section{HAL Id: cea-01481347 https://hal-cea.archives-ouvertes.fr/cea-01481347}

Submitted on 2 Mar 2017

HAL is a multi-disciplinary open access archive for the deposit and dissemination of scientific research documents, whether they are published or not. The documents may come from teaching and research institutions in France or abroad, or from public or private research centers.
L'archive ouverte pluridisciplinaire HAL, est destinée au dépôt et à la diffusion de documents scientifiques de niveau recherche, publiés ou non, émanant des établissements d'enseignement et de recherche français ou étrangers, des laboratoires publics ou privés. 


\title{
Magnetic impurities in nanotubes: From density functional theory to Kondo many-body effects
}

\author{
P. P. Baruselli, ${ }^{1,2,3}$ M. Fabrizio,,${ }^{1,2}$ A. Smogunov,${ }^{4}$ R. Requist, ${ }^{1}$ and E. Tosatti ${ }^{1,2,5}$ \\ ${ }^{1}$ SISSA, Via Bonomea 265, Trieste 34136, Italy \\ ${ }^{2}$ CNR-IOM, Democritos Unitá di Trieste, Via Bonomea 265, Trieste 34136, Italy \\ ${ }^{3}$ Institut für Theoretische Physik, Technische Universität Dresden, 01062 Dresden, Germany \\ ${ }^{4}$ CEA, IRAMIS, SPCSI, F-91191 Gif-sur-Yvette Cedex, France \\ ${ }^{5}$ ICTP, Strada Costiera 11, Trieste 34014, Italy
}

(Received 6 September 2013; revised manuscript received 25 November 2013; published 17 December 2013)

\begin{abstract}
Low-temperature electronic conductance in nanocontacts, scanning tunneling microscopy (STM), and metal break junctions involving magnetic atoms or molecules is a growing area with important unsolved theoretical problems. While the detailed relationship between contact geometry and electronic structure requires a quantitative ab initio approach such as density functional theory (DFT), the Kondo many-body effects ensuing from the coupling of the impurity spin with metal electrons are most properly addressed by formulating a generalized Anderson impurity model to be solved with, for example, the numerical renormalization group (NRG) method. Since there is at present no seamless scheme that can accurately carry out that program, we have in recent years designed a systematic method for semiquantitatively joining DFT and NRG. We apply this DFT-NRG scheme to the ideal conductance of single wall $(4,4)$ and $(8,8)$ nanotubes with magnetic adatoms ( $\mathrm{Co}$ and $\mathrm{Fe}$ ), both inside and outside the nanotube, and with a single carbon atom vacancy. A rich scenario emerges, with Kondo temperatures generally in the Kelvin range, and conductance anomalies ranging from a single channel maximum to destructive Fano interference with cancellation of two channels out of the total four. The configuration yielding the highest Kondo temperature (tens of Kelvins) and a measurable zero-bias anomaly is that of a Co or Fe impurity inside the narrowest nanotube. The single atom vacancy has a spin, but a very low Kondo temperature is predicted. The geometric, electronic, and symmetry factors influencing this variability are all accessible, which makes this approach methodologically instructive and highlights many delicate and difficult points in the first-principles modeling of the Kondo effect in nanocontacts.
\end{abstract}

DOI: 10.1103/PhysRevB.88.245426

PACS number(s): 73.63.Rt, 72.15.Qm, 73.23.Ad, 73.63.Fg

\section{INTRODUCTION}

When the contact between two metals is reduced to the ultimate monoatomic limit - a geometry realized in mechanical break junctions, ${ }^{1}$ but also in $\mathrm{STM}^{2}$ - the electrical conductance can be satisfactorily understood and calculated by applying Landauer's standard ballistic formalism ${ }^{3}$ to an equally standard $a b$ initio electronic structure calculation of the nanocontact; ${ }^{4}$ for an alternative formulation, see Ref. 5. However, when a magnetic atom (such as Co) or magnetic molecule (such as $\mathrm{Cu}$-phthalocyanine) bridges two nonmagnetic metallic leads, the conductance reflects the presence of the impurity spin and its Kondo screening. The characteristic Kondo signature is a low-voltage conductance peak, or dip, present with or without a magnetic field and generally referred to as a zero-bias anomaly. ${ }^{6-8}$

The zero-bias anomaly is determined by the electronic structure of the nanocontact. Given the atomic nature of a nanocontact, as opposed to the smoothness of mesoscopic contacts such as quantum dots, ${ }^{9}$ a quantitative $a b$ initio approach is mandatory to represent the geometry-dependent electronic structure, the local spin density, etc., in realistic detail. That information is available, albeit approximately, from spin-polarized density functional theory (DFT) calculations but comes at the price of breaking spin-rotational symmetry. Spontaneous spin-rotational symmetry breaking does indeed occur in infinite magnetic systems, which DFT describes reasonably well, but not in a single magnetic atom, molecule, or dot. As a result, spin-polarized DFT completely misses the Kondo screening of the local magnetic moment by the leads, ${ }^{10}$ thus failing to provide the correct low-temperature low-field conductance and zero-bias anomaly. A full description of the Kondo physics requires instead an explicit many-body technique, such as NRG. ${ }^{11}$ Although promising approximate $a b$ initio based approaches have been proposed, ${ }^{12,13}$ a quantitatively accurate description of Kondo physics has only been achieved with NRG. But due to the complexity of the problem, NRG-type methods cannot handle all the electronic degrees of freedom of a realistic lead-impurity-lead contact geometry and are only practical for highly simplified Anderson impurity models (AIM), ${ }^{14,15}$ whose parameters could only be estimated phenomenologically thus far, leaving us without a quantitative ab initio based method for the prediction of magnetic nanocontact conductance, even at zero temperature, low voltage, and zero field. To be sure, several important discussions are present in the literature where DFT electronic structure calculations have been employed to argue qualitatively for a given impurity spin, and/or where NRG calculations have been used to distinguish the different temperature and field behavior predicted for different spins. ${ }^{16-21}$ What is, however, still needed is an approach where geometrical and orbital complications are included at the outset and connected to subsequent NRG calculations at a quantitative level.

Here, we present an implementation, based on work recently developed in our group, ${ }^{22}$ which attempts to improve this situation by means of a well defined semiquantitative scheme for joining DFT and NRG. The scheme is straightforward. The basic consideration is that a spin-polarized DFT calculation of a magnetic impurity can be regarded as conceptually similar to the mean-field treatment of a generalized AIM. Like the Hartree-Fock solution of the original 
AIM, ${ }^{14}$ it provides a mean-field rationale for the existence of free local moments in transition metal impurities and alloys in a nonmagnetic host metal. Furthermore, AIMs neglect the interactions between conduction electrons, which parallels the underlying assumption of the local density approximation (LDA) and generalized gradient approximation (GGA) that such interactions only modify the band structure parameters, i.e., the host metal can be described by noninteracting quasiparticles with an effective band dispersion. On the basis of this correspondence, we assume there exists an AIM that reproduces, within mean field, the ab initio results for a generic nanocontact. The key point is to select which particular $a b$ initio quantities the AIM mean field should reproduce in order for the AIM itself to provide the best possible description of the low-temperature conductance through the nanocontact. Concerning the internal electronic degrees of freedom of the impurity, the choice is practically mandatory and is dictated by the localized orbitals that are primarily involved in magnetism. For instance, in the case of a transition metal atom, one must assume that the model includes at least the $d$ orbitals (and potentially one $s$ orbital). The choice of conduction channels among the electronic states of the free leads is similarly mandatory and dictated by the requirement that they should share the same symmetry as the impurity states with which they hybridize.

What is not equally straightforward is to find unambiguous criteria determining the impurity-lead hybridization parameters and the interaction terms acting within the impurity. One problem is that the ab initio results are obtained by explicitly breaking spin SU(2) symmetry, whereas the parameters we are seeking belong to a spin-rotationally invariant Hamiltonian. For instance, the spectral density of states of the magnetic impurity, determined using a basis of localized orbitals, has a strong spin splitting and is generally peaked far away from the chemical potential in spin-polarized DFT, making it difficult to reconstruct the spin-rotationally invariant hybridization functions that enter the AIM. Conversely, the alternative possibility of starting from a spin unpolarized calculation would yield too little information about the spin state of the impurity. For example, it would not tell us whether a $\mathrm{d}^{7}$ impurity, say $\mathrm{Co}^{2+}$, in an octahedral environment has a high-spin or low-spin state, namely $S=3 / 2$ or $1 / 2$.

Our approach toward fixing the model parameters is to make use of the additional information contained in the $a b$ initio scattering phase shifts. ${ }^{22}$ Specifically, given a particular nanocontact geometry, one can identify the symmetry-adapted scattering eigenchannels and, for each of them, calculate the scattering phase shifts for any spin projection relative to the direction of the mean-field magnetization. We shall see in Sec. III that these phase shifts together with a few other quantities from the $a b$ initio calculation will allow us to determine an effective AIM relatively unambiguously. The subsequent solution of this model by NRG provides results that we believe are representative of the low-temperature behavior of the realistic nanocontact, including in particular the low-bias conductance anomalies.

The choice of single wall carbon nanotubes for our application was guided not so much by experiment, which is still missing, but rather by the consideration that nanotubes possess well-defined one-dimensional conduction channels.
The system's intrinsic simplicity and robustness makes it an ideal test case for a thorough study. Although a first exploratory preview was recently presented, ${ }^{23}$ here we now provide a full account of the protocol and of its results, including particularly carbon vacancies, which here play the unusual role of magnetic impurities.

The paper is organized as follows. In Sec. II, we present DFT results for $\mathrm{Co}$ and $\mathrm{Fe}$ impurities on $(4,4)$ and $(8,8)$ nanotubes. In Sec. III, we set up the AIM and explain how to fix its parameters. Sections IV and V present our NRG results concerning Kondo behavior, respectively for $\mathrm{Co}$ and Fe impurities. In Sec. VI we report some results about the zero-bias anomalies to be expected in such systems. The additional case of a nanotube vacancy acting as a magnetic impurity is presented in Sec. VII, including both DFT and NRG results. Finally, in Sec. VIII, we draw the main conclusions of this work.

\section{COBALT AND IRON IMPURITIES ON NANOTUBES: DFT STUDY}

\section{A. Electronic structure}

Our study starts with electronic structure calculations, greatly extending previous ones, Refs. 23 and 24 for single $\mathrm{Co}$ or $\mathrm{Fe}$ atoms adsorbed on a metallic single-wall carbon nanotube (SWNT) (see Fig. 1). In order to study the effect of the nanotube curvature, we considered two different nanotubes, $(4,4)$ and $(8,8)$, of different radius. We did not consider a $\mathrm{Ni}$ adatom since, as recently reported, ${ }^{25}$ it generally loses its magnetic moment when adsorbed on carbon nanotubes.

We begin by defining the scattering region, which we take to be a nanotube segment consisting of $N_{C}$ carbon atoms $\left[N_{C}=80,160\right.$ for $(4,4)$ and $(8,8)$ tubes, respectively $]$ and one impurity. For this system, we first carried out standard DFT calculations with periodic boundary conditions and relaxed the positions of all the atoms in the unit cell shown in Fig. 1, except those in the two outermost rings, to improve the convergence toward the infinite tube limit. Calculations were performed with the plane wave package QUANTUM ESPRESSO ${ }^{26}$ within the GGA to the exchange-correlation energy in the parametrization of Perdew, Burke, and Ernzerhof. ${ }^{27}$ The planewave cutoffs were 30 and $300 \mathrm{Ry}$ for the wave functions and the charge density, respectively. Integration over the one-dimensional Brillouin zone was accomplished using $8 k$ points and a smearing parameter of $10 \mathrm{mRy}$. When necessary to test the effects of electron correlations and self-interaction errors, we extended the calculations from GGA to GGA $+U$, including a Hubbard $U$ interaction within the transition metal $d$ orbitals. While this was occasionally important as a check on the stability of the impurity spin state, most results presented below were obtained in the GGA.

Our DFT calculations suggest that in all cases the hollow site is the most stable adsorption configuration. For example, the on-top (external) Co adsorption configuration was about $47 \mathrm{meV}$ higher in energy that the hollow site on the $(4,4)$ SWNT. Nevertheless, we also included in our study the case of a Co adatom adsorbed at the on-top position of a $(4,4)$ SWNT to gain insight into the influence of adsorption site on the magnetic and transport properties of the nanotube. Also, 
$(4,4)$ hollow $(\mathrm{Co}, \mathrm{Fe})$
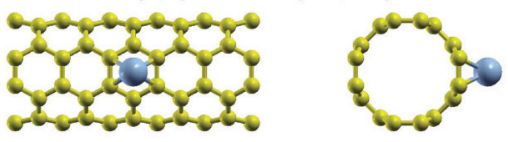

$(4,4)$ top $(\mathrm{Co})$
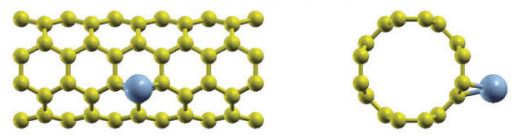

$(4,4)$ inside $(\mathrm{Co}, \mathrm{Fe})$

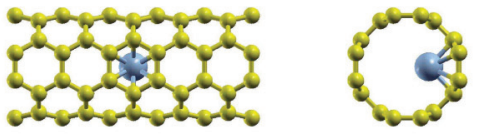

$(8,8)$ hollow $(\mathrm{Co}, \mathrm{Fe})$
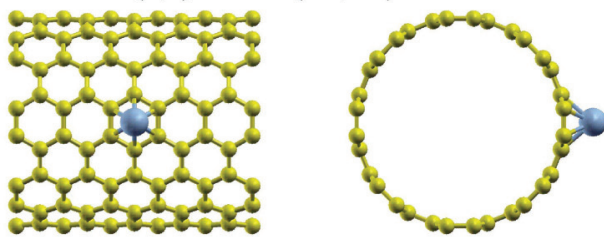

$(8,8)$ inside $(\mathrm{Co}, \mathrm{Fe})$
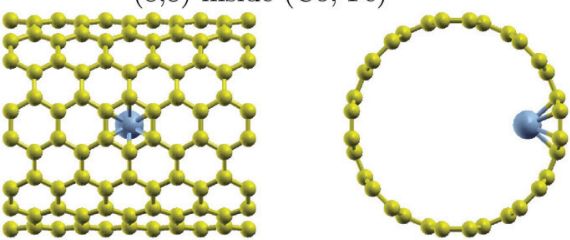

$(4,4)$ Longitudinal Vacancy
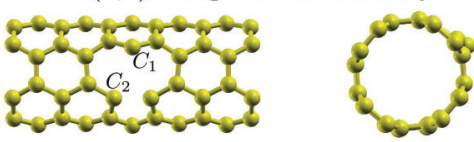

$(4,4)$ Transversal Vacancy
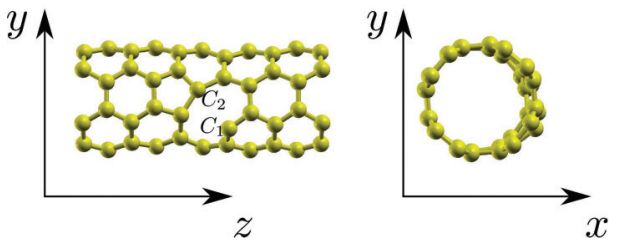

FIG. 1. (Color online) Co and Fe adatoms on single wall carbon nanotubes and a single atom vacancy in two inequivalent relaxed configurations.

although such adsorption geometries are higher in energy, they might still be accessible in experiment. In order to explore the possible role of self-interaction errors, we performed $\mathrm{GGA}+U$ calculations for the selected case of hollow-site Co on the $(4,4) \mathrm{SWNT}$. With a value of $U=2 \mathrm{eV}$ for $d$ orbitals of Co, we did not find meaningful changes of the $S=1 / 2$ state of the Co adatom. Table I summarizes the results of the geometry relaxation for all the systems studied and also reports the total spin magnetic moment for each case. These results compare well with those reported recently by Yagi and co-workers. ${ }^{25}$

Figure 2 presents the projected density of states (PDOS) for the $s$ and $d$ orbitals of the TM adatom. The different curves, labeled in the upper panel, correspond to the character of the orbital. The PDOS shows sharp spin-split peaks corresponding to the magnetic orbitals of the TM atom that will subsequently
TABLE I. Geometry and spin magnetic moment of $\mathrm{Co}$ and $\mathrm{Fe}$ adatoms on different SWNTs. The number of equivalent $\mathrm{C}$ atoms with the same nearest-neighbor distance to the adatom is given in parentheses; in the case of vacancies, the distance of the lone $C_{1}$ atom to its nearest neighbors is given. The last column gives the total spin magnetic moment obtained by integrating the spin magnetization over the whole unit cell.

\begin{tabular}{lcc}
\hline \hline Configuration & TM-C dist. $(\AA)$ & $\mu\left(\mu_{B}\right)$ \\
\hline$(4 \times 4)$ Co hollow & $2.07(4), 2.32(2)$ & 1.26 \\
$(4 \times 4)$ Co hollow, $U=2 \mathrm{eV}$ & $2.08(4), 2.33(2)$ & 1.17 \\
$(4 \times 4)$ Co on-top $(\Delta E=47 \mathrm{meV})$ & $1.99(2), 2.00(1)$ & 1.16 \\
$(4 \times 4)$ Co inside $(\Delta E=193 \mathrm{meV})$ & $2.15(4), 1.94(2)$ & 0.79 \\
$(4 \times 4)$ Co inside, $U=2 \mathrm{eV}$ & $2.19(4), 1.97(2)$ & 1.01 \\
$(4 \times 4)$ Fe hollow & $2.17(4), 2.42(2)$ & 3.40 \\
$(4 \times 4)$ Fe inside $(\Delta E=199 \mathrm{meV})$ & $2.15(4), 1.98(2)$ & 1.84 \\
$(8 \times 8)$ Co hollow & $2.09(4), 2.21(2)$ & 1.32 \\
$(8 \times 8)$ Fe hollow & $2.11(4), 2.23(2)$ & 2.35 \\
$(8 \times 8)$ Co inside $(\Delta E=53 \mathrm{meV})$ & $2.11(4), 2.02(2)$ & 1.18 \\
$(8 \times 8)$ Fe inside $(\Delta E=130 \mathrm{meV})$ & $2.13(4), 2.05(2)$ & 2.15 \\
$(4 \times 4)$ Long. vac. $(\Delta E=0.8 \mathrm{eV})$ & $1.37(2), 2.86(2)$ & 1.05 \\
$(4 \times 4)$ Transversal vacancy & $1.39(1), 1.39(1)$, & 0.89 \\
& $2.64(1), 2.70(1)$ & \\
\hline \hline
\end{tabular}

be used to construct the many-body model Hamiltonian. The crucial element here is symmetry. All the TM orbitals can be classified according to their symmetry as follows. In both hollow and on-top geometries, there is a mirror plane $x y$ through the TM atom and orthogonal to the nanotube (see Fig. 1). The states are therefore either even $(e)$ or odd $(o)$ with respect to the corresponding reflection operation. For the hollow-adsorption site, there is in addition the symmetry plane $x z$. We can assign therefore an extra index $s$ (symmetric) or $a$ (antisymmetric) to states which are even or odd with respect to this additional symmetry plane. As an example, consider the Co atom adsorbed at the hollow site of the $(4,4)$ nanotube (upper panel of Fig. 2). In this case, there is only one magnetic orbital, $d_{x z}$, which has the $\{o, s\}$ symmetry and is singly occupied by a spin up electron in our DFT calculations. All other $d$ orbitals are fully occupied and therefore irrelevant for low-temperature physics, including the $d_{x y}$ orbital, which, partially empty in the spin down channel, becomes almost fully occupied when a finite $U=2 \mathrm{eV}$ is introduced in the calculation (see the second panel from the top in Fig. 2). This concludes the analysis of the relevant impurity orbitals and their symmetry.

The next step is to identify the nanotube conduction channels carrying the electrons which will scatter on the magnetic impurity. This is done by examining the electronic structure of the infinite, impurity-free nanotube. Figure 3 shows the band structure of $(4,4)$ and $(8,8)$ carbon nanotubes with, in both cases, two conduction bands crossing the chemical potential. One is symmetric and the other antisymmetric with respect to the mirror $x z$ plane. We label them as $s$ and $a$ in accordance with the above notation. Each of these two bands has leftand right-moving states, $\phi_{l}$ and $\phi_{r}$, which can be combined to form even and odd combinations, $\phi_{e / o}=\left(\phi_{l} \pm \phi_{r}\right) / \sqrt{2}$. The four resulting conduction channels, which can be labeled by 


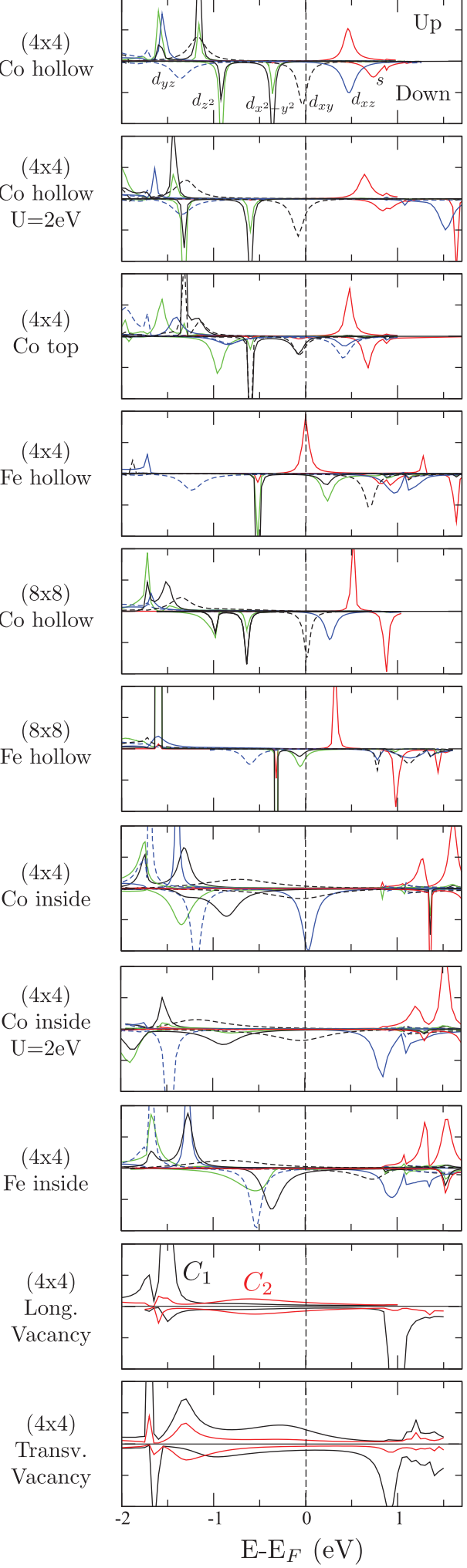

FIG. 2. (Color online) PDOS on the impurity atomic orbitals. The curves on the upper panel are marked by the corresponding atomic character. Dashed vertical lines indicate the position of the Fermi level. We do not show the case of Co and Fe inside the $(8,8)$ SWNT, but it is qualitatively similar to the case inside the $(4,4)$ SWNT. In the case of vacancies, the total PDOS on $2 s$ and $2 p$ orbitals of atoms denominated $C_{1}$ (the lone atom) and $C_{2}$ (one of the other two atoms originally close to the removed atom, see Fig. 1) is given.
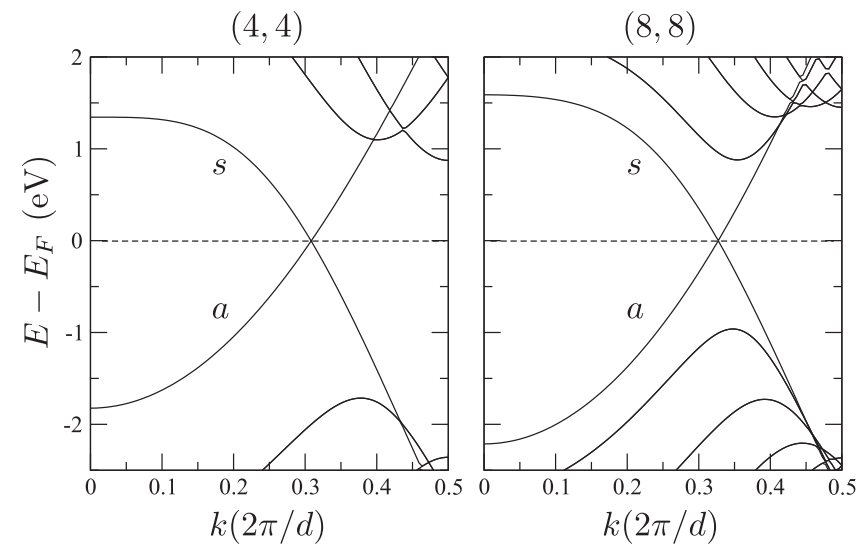

FIG. 3. Electronic bands of pure $(4,4)$ and $(8,8)$ SWNTs. The two bands crossing the Fermi energy are labeled as $s$ (symmetric) and $a$ (antisymmetric), which reflects their symmetry with respect to the mirror plane $x z$ (see Fig. 1).

the pair $\{e / o, s / a\}$, identify the four scattering channels that will couple to impurity orbitals of same symmetry.

\section{B. Transmission function and phase shifts from density functional calculations}

The main physical property of interest to us is the linear electrical conductance near zero bias of the nanotube with a single magnetic impurity. Within the mean-field DFT scheme, which is the initial stage of our approach, the linear response ballistic conductance is given by the Landauer-Buttiker formula, $G=\left(G_{0} / 2\right) T\left(E_{F}\right)$, where $T\left(E_{F}\right)$ is the total electron transmission at the Fermi level and $G_{0}=2 e^{2} / h$ is the conductance quantum. In our collinear spin-polarized calculations, the total transmission is just the sum of the two independent spin channels, $T\left(E_{F}\right)=\sum_{\sigma} T^{\sigma}\left(E_{F}\right)$. The transmission function in each spin channel is given by the trace (suppressing the spin index and the energy argument), $T=\operatorname{tr}\left(\mathbf{t}^{\dagger} \mathbf{t}\right)$, where $t_{i j}$ is in our case the $(2 \times 2)$ matrix of transmission amplitudes with $i, j=s$ or $a$. For the hollow adsorption site, this matrix is diagonal since scattering conserves reflection symmetry in the $x z$ plane, and therefore $T=\left|t_{s}\right|^{2}+\left|t_{a}\right|^{2}$, where $t_{s / a}$ are the transmission amplitudes for the two independent channels. It should be stressed here that the mean-field transmission and the associated conductance are simply an intermediate calculational step and by no means represent our final conductance result, which will follow the NRG study.

In order to calculate the transmission amplitudes, we take the unit cell of Fig. 1 as the scattering region and smoothly attach semi-infinite carbon nanotubes to both sides. The transmission and reflection amplitudes of such an open system are then calculated using a wave function matching approach ${ }^{28}$ implemented in the PWCOND code, which is part of the QUANTUM ESPRESSO package.

We present in Fig. 4 spin-dependent transmission functions for all cases under consideration. Around the Fermi energy, the total transmission per spin has a maximum value of two, corresponding to the two available electron bands. As a function of energy, the transmission curves display several sharp dips in correspondence with the peaks of the adatom DOS, cf. Fig. 2. Here, the transmission drops from the ideal 


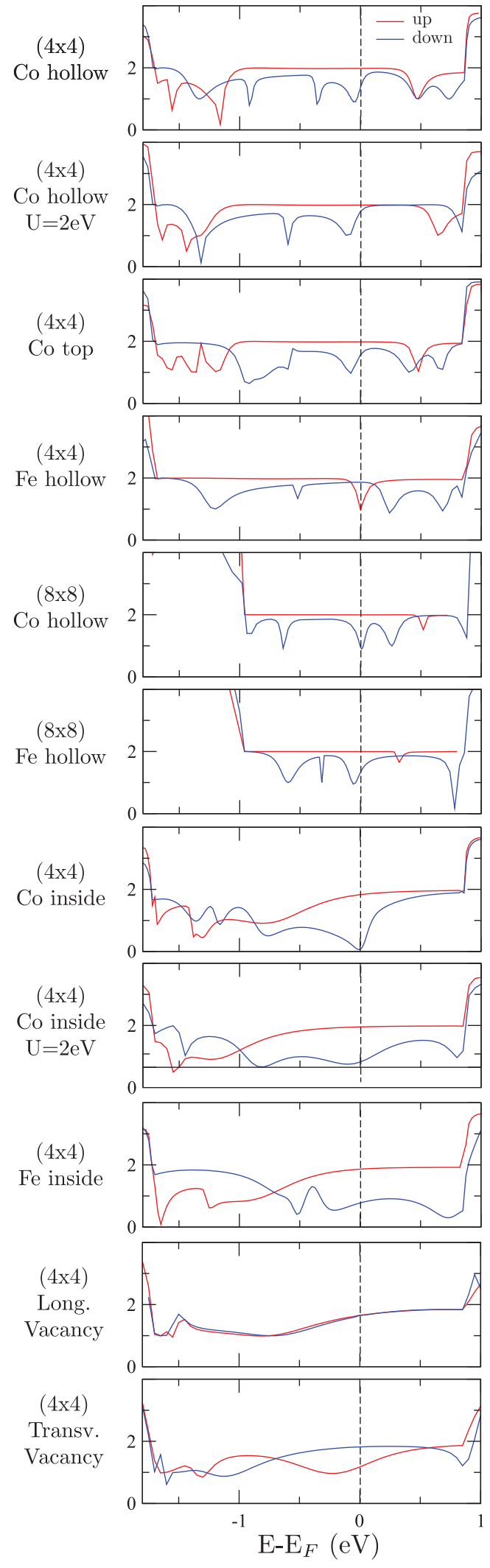

FIG. 4. (Color online) Spin-polarized transmission functions for the same cases as those in Fig. 2.

value of 2 to 1 since one of the channels, $s$ or $a$, gets suppressed due to destructive interference between pathways going straight along the nanotube and those passing through
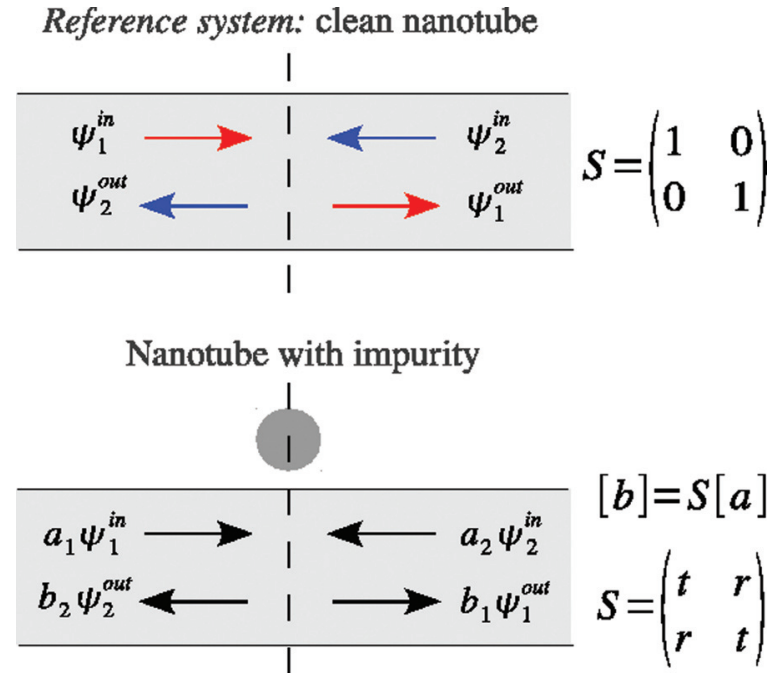

FIG. 5. (Color online) Schematic picture explaining the calculation of even and odd phase shifts $\delta_{e / o}$. They appear after diagonalizing of the scattering matrix $S$. The clean nanotube without impurity is the reference system defining the incoming and outgoing waves with respect to which the scattering matrix is calculated when the impurity is introduced.

the adatom orbital of the same symmetry. If a DOS peak for one spin polarization occurs very close to the Fermi energy, then the mean-field conductance of two spin channels differs significantly. That is the case, for example, for hollow site Co on the $(8,8)$ nanotube and hollow site Fe on the $(4,4)$ nanotube. Of course, these DFT results are expected to be significantly altered by many-body effects in the low-temperature regime (see discussion below).

The crucial quantities characterizing the scattering of conduction band states on the impurity are the scattering phase shifts. They are obtained by diagonalizing the unitary $S$ matrix relating the amplitudes of outgoing and incoming scattering waves. In our case of a nanotube with two bands at the Fermi energy, the $S$ matrix (for each spin channel) will be a $(4 \times 4)$ matrix, two states provided by the left half of the nanotube and two by the right one. Figure 5 shows schematically how the phase shifts are calculated. Let us consider, for example, the case of the hollow adsorption site. Here, by symmetry, the $s$ and $a$ channels do not mix so that the $S$ matrix factorizes into two independent $(2 \times 2)$ blocks. We define as reference system a clean nanotube without the impurity, so that the unperturbed $S$ matrix is just the unit matrix. When the impurity is introduced at the hollow side, $S$ transforms into

$$
S=\left(\begin{array}{ll}
t & r \\
r & t
\end{array}\right)
$$

where $t$ and $r$ are transmission and reflection amplitudes, respectively. The matrix is symmetric due to the mirror $x y$ symmetry plane. Diagonalizing $S$, we obtain

$$
U^{\dagger} S U=\left(\begin{array}{cc}
e^{2 i \delta_{e}} & 0 \\
0 & e^{2 i \delta_{o}}
\end{array}\right), \quad U=\frac{1}{\sqrt{2}}\left(\begin{array}{cc}
1 & 1 \\
1 & -1
\end{array}\right),
$$

which reveals the two phase shifts, $\delta_{e}$ and $\delta_{o}$, corresponding to even and odd eigenchannels as given by the columns of the unitary matrix $U$. For each symmetry channel, $s$ or 

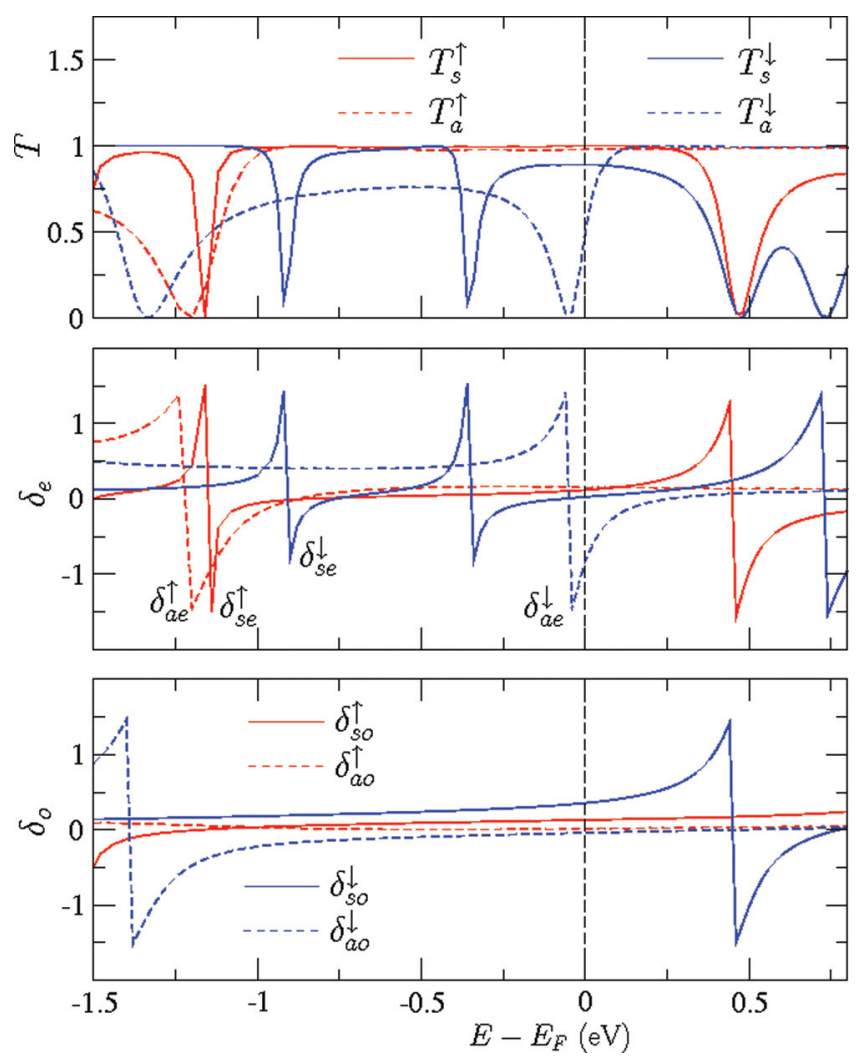

FIG. 6. (Color online) Spin-polarized transmission eigenvalues (top) and even (middle), and odd (bottom) phase shifts, $\delta_{e / o}$, for a Co adatom on $(4,4)$ SWNT (hollow site). Solid and dashed lines correspond to symmetric and antisymmetric channels (with respect to the $x z$ plane). The curves for spin-up and spin-down polarizations are shown in red and blue colors, respectively.

$a$, we thus obtain two phase shifts, even and odd. From Eqs. (1) and (2), one can easily verify the following wellknown relationship between phase shifts and transmission and reflection probabilities:

$$
|t|^{2}=\cos ^{2}\left(\delta_{e}-\delta_{o}\right), \quad|r|^{2}=\sin ^{2}\left(\delta_{e}-\delta_{o}\right) .
$$

On the other hand, the phase shifts can also be related to the extra DOS (of the same symmetry), $\Delta \rho$, induced by the impurity via the Friedel sum rule:

$$
\Delta \rho_{i}(E)=\frac{1}{\pi} \frac{d \delta_{i}(E)}{d E}, \quad i=e, o .
$$

The DFT phase shifts thus fully characterize the link between the electronic structure and the transport properties of the system. We note that the two channels, $s$ and $a$, get mixed for the on-top adsorption site since the symmetry plane $x z$ is missing, and one simply has two even and two odd phase shifts.

As an example, we present in Fig. 6 the transmission functions and phase shifts for the case of hollow site Co on the $(4,4)$ nanotube. Transmission functions for both spin channels and both symmetries, $s$ and $a$, are plotted on the upper panel while the even and odd phase shifts are shown on the middle and lower panels, respectively. Since the phase shifts are defined modulo $\pi$, we choose to plot them on the interval $[-\pi / 2, \pi / 2]$. One can see from the figure that all the dips in transmission are associated with abrupt changes (by the value $\sim \pi$ ) in either even or odd phase shifts of the same symmetry, in agreement with Eq. (3).

These sharp features in the phase shifts are directly related to PDOS peaks of the same symmetry (see Fig. 2, upper panel), as implied by the Friedel sum rule, Eq. (4). For example, in spin-down transmission of $s$ symmetry, there are two dips at energies around 0.5 and $0.75 \mathrm{eV}$. The corresponding sharp features in the odd and even phase shifts derive, respectively, from the Co $d_{x z}$ and $s$ orbitals, hybridized with conduction electrons of the nanotube. The phase shifts calculated within the DFT approach are now ready to play the subsequent central role in generating the parameters for the AIMs.

\section{ANDERSON MODELS AND THEIR HARTREE FOCK PHASE SHIFTS}

In this section, we describe the method used to build effective AIMs starting from $a b$ initio calculations of the nanotube with a transition metal impurity. The scattering calculations described in the previous section yield different phase shifts for spin up and spin down conduction electrons, which is of course an artifact of spin-rotational symmetry breaking. The root of the problem is that a broken-symmetry $a b$ initio calculation misses quantum fluctuations between mean-field solutions with magnetization oriented in different directions, a process intrinsic to the Kondo effect that restores spin-rotational symmetry. In the context of AIMs, a physically transparent way of starting from a mean-field solution with a preformed local moment and subsequently including quantum fluctuations is the Anderson-Yuval-Hamann path integral approach. ${ }^{29,30}$ In the same way, we could, in principle, restore spin symmetry by building quantum fluctuations on top of the $a b$ initio calculation. However, since our goal is to go from the $a b$ initio data to the final result by way of a model Hamiltonian, we shall instead exploit the close analogy, mentioned in the introduction, between a spin-polarized DFT calculation and the mean-field solution of a generalized AIM. Namely, we adjust the model parameters so that the scattering phase shifts of the AIM, at the mean-field level, exactly reproduce the $a b$ initio phase shifts. The model Hamiltonian thus obtained will provide a faithful low-energy representation of the actual nanocontact if the quantum fluctuations in the model are in some sense similar to the local quantum fluctuations of the impurity. All exact symmetries including spin-rotational symmetry are restored in the final step of our calculations, when the model Hamiltonian is solved with the NRG method.

Since the NRG method is numerically intensive, we will only be able to deal with a very limited number of channels and symmetries. Accordingly, our description of the electronic structure of the clean metallic tube will be necessarily crude, encompassing two conduction bands only. These bands are assumed to have a linear dispersion $E(k)= \pm v\left|k-k_{F}\right|$ around the Fermi energy (FE), giving rise to a constant density of states (DOS) $\rho_{0}=1 / \pi v$ at the FE. As discussed in Sec. II, there are four scattering channels (corresponding to the symmetries $e s, e a, o s, o a$ ), each with the same DOS at the FE. Each channel couples to the impurity orbitals with the same symmetry. It should be noted here that the neglect of all other nanotube subbands restricts our treatment to SWNTs 
TABLE II. Valence orbitals of $\mathrm{Fe}$ and $\mathrm{Co}$ and their symmetries (e/o: even-odd with respect to the $x y$ plane; $s / a$ : symmetricantisymmetric with respect to the $x z$ plane; $z$ is the axis of the tube). On the left, hollow configuration, on the right, on-top configuration.

\begin{tabular}{ccccc}
\hline \hline & Hollow & & & On-top \\
\hline & $s$ & $a$ & & \\
\cline { 2 - 5 }$e$ & $d_{z^{2}}, d_{x^{2}-y^{2}}, s$ & $d_{x y}$ & $e$ & $d_{z^{2}}, d_{x^{2}-y^{2}}, d_{x y}, s$ \\
$o$ & $d_{x z}$ & $d_{y z}$ & $o$ & $d_{x z}, d_{y z}$ \\
\hline \hline
\end{tabular}

of smallest radius, where these subbands are sufficiently far from the Fermi level. This obstructs, in particular, any attempt to extrapolate towards the infinite radius limit, i.e., graphene, where all subbands coalesce at the FE. The impurity orbitals that will be considered here are those in the valence shell of the TM atom, namely, the five $3 d$ orbitals and the $4 s$ orbital, whose symmetry properties are listed in Table II. When the impurity is in the hollow site, the parities with respect to both reflection planes are good quantum numbers, and we can classify electronic states (both of the tube and the adatom) accordingly. When instead the impurity is in the on-top position, only $e / o$ is a good quantum number, since symmetric and antisymmetric conduction states are mixed together. Here, the problem is somewhat harder to treat; we will briefly illustrate this case later, while, in what follows, we shall always refer to the hollow configuration, which is anyway the lowest in energy.

Our general AIM includes therefore four scattering channels, $i=e s, e a, o s, o a$, and six impurity orbitals, $a=1, \ldots, 6$; hence, it is of the form

$$
\begin{aligned}
H= & \sum_{i k \sigma}\left[\epsilon_{k} c_{i k \sigma}^{\dagger} c_{i k \sigma}+\sum_{a} V_{i k, a}\left(c_{i k \sigma}^{\dagger} d_{a \sigma}+\text { H.c. }\right)\right] \\
& +\sum_{i k k^{\prime} \sigma} t_{i, k k^{\prime}} c_{i k \sigma}^{\dagger} c_{i k^{\prime} \sigma}+H_{\mathrm{imp}},
\end{aligned}
$$

where $c_{i k \sigma}^{\dagger}$ creates a spin $\sigma$ electron in channel $i$ with momentum $k$ along the tube, $d_{a \sigma}^{\dagger}$ a spin $\sigma$ electron in the orbital $a$ of the impurity. $V_{i k, a}$ is the hybridization matrix element between conduction and impurity orbitals, which is finite only if they share the same symmetry according to Table II, while $t_{i, k k^{\prime}}$ describes a local scalar potential felt by the conduction electrons because of the translational symmetry breaking caused by the impurity. $H_{\text {imp }}$ includes all terms that involve only the impurity orbitals, which we can write as

$$
H_{\mathrm{imp}}=\sum_{a \sigma}\left(\epsilon_{a} n_{a}+U_{a} n_{a \uparrow} n_{a \downarrow}\right)+\sum_{a<b} U_{a b} n_{a} n_{b}+H_{\mathrm{Hund}},
$$

where $n_{a \sigma}=d_{a \sigma}^{\dagger} d_{a \sigma}, n_{a}=\sum_{\sigma} n_{a \sigma}$ and $H_{\text {Hund }}$ contains all interorbital interaction terms that in the isolated atom would give rise to Hund's rules. Since the degeneracy among the $d$ orbitals is fully removed in our scattering geometry, we will only take the first Hund's rule into account thus writing

$$
H_{\mathrm{Hund}}=\sum_{a<b} J_{a b} \mathbf{S}_{a} \cdot \mathbf{S}_{b},
$$

where $J_{a b}<0$, favoring a ferromagnetic correlation among the spin densities $\mathbf{S}_{a}$ of the different orbitals.

The parameters in this Hamiltonian are so far unknown. As described earlier on, our goal is to establish a direct correspondence between a mean-field solution of this model Hamiltonian, and the detailed DFT calculation of the previous chapter that will allow, even if approximately, the extraction of ab initio based parameters. The mean-field treatment of (5) is quite straightforward. One assumes that

$$
\begin{aligned}
U_{a} n_{a \uparrow} n_{a \downarrow} & \longrightarrow U_{a} \sum_{\sigma}\left\langle n_{a-\sigma}\right\rangle n_{a \sigma}, \\
U_{a b} n_{a} n_{b} & \longrightarrow U_{a b}\left\langle n_{a}\right\rangle n_{b}+U_{a b}\left\langle n_{b}\right\rangle n_{a}, \\
J_{a b} \mathbf{S}_{a} \cdot \mathbf{S}_{b} & \longrightarrow J_{a b}\left\langle\mathbf{S}_{a}\right\rangle \cdot \mathbf{S}_{b}+J_{a b}\left\langle\mathbf{S}_{b}\right\rangle \cdot \mathbf{S}_{a},
\end{aligned}
$$

where $\langle\ldots\rangle$ is the average value, to be determined selfconsistently, with respect to the Hartree-Fock Slater determinant. It follows that the Hartree-Fock Hamiltonian describes noninteracting orbitals, each one characterized by an effective spin-dependent energy

$$
\begin{aligned}
\epsilon_{a \sigma}^{*}= & \epsilon_{a}+U_{a}\left\langle n_{a-\sigma}\right\rangle \pm \frac{1}{4} \sum_{b} J_{a b}\left\langle n_{b \uparrow}-n_{b \downarrow}\right\rangle \\
& +\sum_{b} U_{a b} n_{b},
\end{aligned}
$$

where the plus/minus sign refers to $\sigma=\uparrow / \downarrow$. Each channel $i$ scattering off the impurity region acquires a spin-dependent phase shift $\delta_{i \sigma}$ caused by the potential term $t_{i, k k^{\prime}}$ as well as the hybridization with the localized orbitals. We assume that $t_{i, k k^{\prime}}$ alone would produce a scattering phase shift $\phi_{i}$. It follows that, if we concentrate on the region close to the chemical potential where the DOS is constant, the total phase shift satisfies the equation

$$
\tan \delta_{i \sigma}=\tan \phi_{i}+\sum_{a} \frac{\Gamma_{i a}}{\epsilon_{a \sigma}^{*}},
$$

where

$$
\Gamma_{i a}=\pi \sum_{k} V_{i k, a}^{2} \delta\left(\epsilon_{k}-\epsilon_{F}\right)
$$

is the hybridization width at the Fermi energy. The ab initio knowledge of the phase shifts $\delta_{i \uparrow}$ and $\delta_{i \downarrow}$ allows us to fix only two parameters in Eq. (9).

When the channel $i$ is coupled to a single orbital, one could fix $\phi_{i}$ and $\Gamma_{i a}$ should $\epsilon_{a \sigma}^{*}$ be known. If the ab initio PDOS of the impurity orbital $a$ with spin $\sigma$ has a well pronounced peak at some energy, it is reasonable to identify the latter with $\epsilon_{a \sigma}^{*}$. This is generally the case, however, in some instances the PDOS of the impurity orbital has a long tail that extends up to the edge of the lowest subband, where not only the conduction electron DOS deviates strongly from the constant FE value $\rho_{0}$, displaying a characteristic one-dimensional van Hove singularity, but other subbands also contribute to the hybridization. In such situations, the assumptions underlying Eq. (9) are no longer valid, and one should in principle take into account the energy dependence of the phase shifts and not just their value at the chemical potential. This is feasible but makes the calculations much more involved. Instead, we adopted a simplified route consisting of keeping just the lowest 
subband, assuming a constant DOS $\rho_{0}$ and fixing $\epsilon_{a \sigma}^{*}$ as the energy where the integrated PDOS is about one half. This assumption is justified only so long as the final results do not depend strongly on the precise choice of $\epsilon_{a \sigma}^{*}$, which we will verify a posteriori.

Having fixed $\epsilon_{a \sigma}^{*}, \phi_{i}$, and $\Gamma_{i a}$, we now need to determine $\epsilon_{a}$, $U_{a}$, and $J_{a b}$ - still too many parameters. One can reduce them by assuming that $J_{a b}$ is constant within the $d$ shell $\left(J_{a b}=J\right)$ and that $J_{s d}$ is the same for all $d$ orbitals. Another reasonable assumption, which can be verified directly in the $a b$ initio calculation, is that the magnetization $M_{s}$ of the $s$ orbital is negligible, so that its spin splitting is controlled by the total $d$ magnetization $M_{d}$ through $J_{s d} M_{d}$, see Eq. (8). This fixes $J_{s d}$. Then, $J$ can be determined through the spin-splitting $J M_{d}+$ $J_{s d} M_{s} \simeq J M_{d}$ of the fully occupied/empty $d$ orbitals. The knowledge of $J$ and $J_{s d}$ allows us to determine $U_{a}$ of the partially filled $d$ orbitals. Finally, we fix $U_{a b}$ by

$$
U_{a b}=U_{a v}-\frac{5}{4} J,
$$

where $U_{a v}$ is the average of all $U_{a}$. Equation (10) holds for an isolated atom; ${ }^{31}$ we assume it remains approximately valid when the degeneracy of the $d$ orbitals is broken, since it involves an average.

We emphasize that $M_{s}, M_{d}$, and $\left\langle n_{a \sigma}\right\rangle$ as well as $\phi_{i}$ and $\Gamma_{i a}$ depend implicitly on the various parameters $J, J_{s d}, U_{a}$ and $U_{a b}$, so that fixing them actually requires solving the HartreeFock equations self-consistently. Once this program has been accomplished, all AIM parameters are determined in such a way that the mean field reproduces the $a b$ initio phase shifts and the energetic position of the impurity levels.

The above scheme works when each channel $i$ is coupled to a single orbital $a$. However, for Fe on the $(4,4)$ nanotube the es channel is coupled to two orbitals, $d_{z^{2}}$ and $s$. In this case, further assumptions are required to determine the AIM parameters, which we shall discuss later.

The AIM Hamiltonian (5) constructed in this way, already greatly simplified with respect to the full physical situation represented by the ab initio starting point, still has too many degrees of freedom to be treated by accurate many-body techniques such as NRG. Since our final goal is to describe the low-temperature and low-bias properties, we can neglect orbitals that are either fully occupied or empty within DFT, provided the energy scale relevant for magnetic quantum fluctuations, i.e., the Kondo temperature, is much smaller than the energy required to excite electrons/holes from those orbitals. This condition has to be verified a posteriori, but we anticipate that it actually holds. Discarding such inert orbitals, namely assuming that they are decoupled from the conduction electrons and just contribute to the scalar potential $t_{i, k k^{\prime}}$ in Eq. (5), it turns out that the number of active orbitals is two for a Co impurity (one of them, $d_{x z}$, being half filled and magnetic and the other one, $d_{x y}$, almost filled). The number of relevant orbitals is instead three for Fe on an $(8,8)$ tube where $d_{x z}$ and $d_{x y}$ are magnetic, and $d_{z^{2}}$ almost filled, and four in the case of $\mathrm{Fe}$ on the $(4,4)$ tube, where besides the three orbitals of the $(8,8)$ case also the $4 s$ orbital is found to be partially occupied in DFT. In conclusion, for Co, only the $o s$ and ea channels are effectively hybridized with the impurity orbitals $d_{x z}$ and $d_{x y}$, respectively. In the case of $\mathrm{Fe}$, we must additionally include the hybridization between the es channel and the $d_{z^{2}}$ orbital in the case of the $(8,8)$ tube, and the $d_{z^{2}}$ and $s$ orbitals in the case of the $(4,4)$ tube.

\section{CO INSIDE AND OUTSIDE NANOTUBES: RESULTS}

In the previous section, we showed how to derive Anderson impurity models that should correctly capture the low-temperature nanotube transport properties. We refer to Appendix A for details about how to solve these models and to Appendix B for all DFT-GGA quantities relevant for the different cases. All AIM parameters are listed in Table III. In this section, we present the actual solution in the case of a Co impurity absorbed inside or outside a nanotube. This case will also serve to explore and expose the possible magnitude of errors introduced by the inaccuracies of the starting DFT electronic structure, generally attributed to incomplete cancellation of self-interactions. It was found that these errors may be important in delicate cases where different orbitals compete, calling for additional care at the outset of the calculations.

\section{A. Co outside a $(4,4)$ tube, hollow site}

According to DFT, in this geometry, Co is in a configuration very close to $3 d^{9} 4 s^{0}$, and hence with spin $S=1 / 2$. In particular, it has only one truly magnetic orbital, $d_{x z}$, coupled to the os conduction channel, along with the almost fully occupied, i.e., only partially magnetized, $d_{x y}$ orbital coupled to the ea channel. All other orbitals are assumed to be inactive, and the effective AIM thus comprises two orbitals, each coupled to its own separate channel. The two impurity states, however, are coupled to one another by a ferromagnetic exchange $J$ and an interorbital Hubbard repulsion $U_{12}$. The two remaining channels $o a$ and $e s$ are free (apart from potential scattering) since they do not couple to any magnetic orbital. Because it is somewhat unusual, the $S=1 / 2$ spin state of nanotube-adsorbed Co required some checking, to avert the possibility that it might arise as an artifact of, for instance, GGA self-interaction errors. We found in fact that $S=1 / 2$ for Co on $(4,4)$ is stable against removal of self interaction. Using, for example, GGA $+U$ with $U=2 \mathrm{eV}$, we obtained qualitatively the same result as for pure GGA: orbital $d_{x z}$ is magnetic, orbital $d_{x y}$ is almost fully occupied, and orbital $s$ is empty. All relevant parameters are listed in Tables III and V.

First of all, we performed an NRG run for each active channel ignoring their mutual coupling, that is setting $J=$ $U_{12}=0$. In this way, we found the phase shifts $\delta_{o s}$ and $\delta_{e a}$ (indicated as $\delta_{\mathrm{NRG}}$ in Table III) and, together with $\delta_{e s} \equiv \phi_{e s}$ and $\delta_{o a} \equiv \phi_{o a}$, the zero-bias conductance for each channel using Eq. (A1) ( $g_{\mathrm{NRG}}$ in Table III). It turns out that this first-run phase shift is almost $\pi / 2$ for the $o s$ channel, as expected for a Kondo channel close to particle-hole symmetry, while the $e a$ channel suffers only a negligible phase shift, as the $d_{x y}$ orbital is almost fully occupied and potential scattering is negligible. We also estimated a first-run Kondo temperature $T_{K}$ of the order of $3 \mathrm{~K}$ for the Kondo channel os.

We then performed a successive NRG run with both channels, now coupled by $J$ and $U_{12}$. The Kondo temperature decreased to $T_{K} \simeq 0.6 \mathrm{~K}$ (this is indicated as $T_{K}$ in Table III). The decrease is appreciable although not dramatic since $d_{x y}$ is almost fully occupied. We now have a concrete example 
TABLE III. Recapitulative table of the parameters used in the Anderson Hamiltonian for different configurations of Fe and Co on both $(4,4)$ and $(8,8)$ tubes; for $\mathrm{Co}(4,4)$ in the hollow position (both outside and inside), a calculation with GGA $+U(U=2 \mathrm{eV})$ is also reported. For each case, we report the orbitals that are involved in transport and their symmetries, the Anderson parameters (the broadening $\Gamma$, the level energy $\epsilon$, the Hubbard repulsion $U$, the Hund exchange $J$, and the interorbital Hubbard repulsion $U_{12}$ ), the results of the solution of the Anderson model (the Kondo temperature $T_{K}$, the NRG phase shift $\delta_{\mathrm{NRG}}$, and the conductance $g_{\mathrm{NRG}}$ in units of $G_{0}$ for each band), and finally, the DFT conductance per band at the Fermi energy $g_{\mathrm{DFT}}=g_{\mathrm{DFT}}^{\uparrow}+g_{\mathrm{DFT}}^{\downarrow}$ for the purpose of comparing with the many-body result.

\begin{tabular}{|c|c|c|c|c|c|c|c|c|c|c|c|}
\hline System & Orb. & Sym. & $\Gamma$ & $\epsilon$ & $\mathrm{U}$ & $\mathrm{J}$ & $U_{12}$ & $T_{K}(\mathrm{~K})$ & $-\delta_{\mathrm{NRG}}$ & $g_{\mathrm{NRG}}$ & $g_{\text {DFT }}$ \\
\hline \multirow[t]{2}{*}{$\mathrm{Co} /(4,4)$} & $d_{x z}$ & os & 0.087 & -3.91 & 2.42 & 1.21 & 1.08 & 0.6 & $0.45 \pi$ & 0.03 & 0.95 \\
\hline & $d_{x y}$ & $e a$ & 0.051 & -4.34 & 2.77 & $\ldots$ & $\ldots$ & & $0.11 \pi$ & 0.93 & 0.72 \\
\hline$\overline{\mathrm{Co}} /(4,4)$ & $d_{x z}$ & os & 0.087 & -6.02 & 3.67 & 1.27 & 2.09 & 0.001 & $0.50 \pi$ & 0.00 & 0.99 \\
\hline $\mathrm{U}=2$ & $d_{x y}$ & $e a$ & 0.059 & -6.46 & 3.69 & $\ldots$ & $\ldots$ & & $0.05 \pi$ & 0.98 & 0.90 \\
\hline$\overline{\mathrm{Co} /(4,4)}$ & $d_{y z}$ & $o$ & 0.076 & -3.41 & 2.43 & 1.25 & 0.75 & 3 & $0.50 \pi$ & 0.01 & 0.99 \\
\hline on-top & $d_{x y}$ & $e$ & 0.059 & -3.54 & 2.20 & $\ldots$ & $\ldots$ & & & 1 & 0.78 \\
\hline \multirow{2}{*}{$\begin{array}{l}\mathrm{Co} /(4,4) \\
\text { inside }\end{array}$} & $d_{x z}$ & os & 0.079 & -2.62 & 2.37 & 1.07 & 0.15 & & $0.22 \pi$ & 0.59 & 0.49 \\
\hline & $d_{x y}$ & $e a$ & 0.380 & -2.19 & 1.99 & $\ldots$ & $\ldots$ & 600 & $0.41 \pi$ & 0.14 & 0.46 \\
\hline \multirow{2}{*}{$\begin{array}{l}\mathrm{Co} /(4,4) \\
\text { inside } \mathrm{U}=2\end{array}$} & $d_{x z}$ & os & 0.086 & -6.52 & 4.73 & 1.44 & 1.62 & $10^{-5}$ & $0.50 \pi$ & 0.01 & 0.88 \\
\hline & $d_{x y}$ & $e a$ & 0.365 & -4.10 & 2.11 & $\ldots$ & $\ldots$ & & $0.28 \pi$ & 0.38 & 0.51 \\
\hline \multirow[t]{2}{*}{$\overline{\mathrm{Co} /(8,8)}$} & $d_{x z}$ & os & 0.058 & -3.98 & 2.49 & 1.15 & 1.02 & 0.1 & $0.45 \pi$ & 0.04 & 0.95 \\
\hline & $d_{x y}$ & $e a$ & 0.038 & -3.98 & 2.43 & $\ldots$ & $\ldots$ & & $0.09 \pi$ & 1.00 & 0.53 \\
\hline \multirow{2}{*}{$\begin{array}{l}\mathrm{Co} /(8,8) \\
\text { inside }\end{array}$} & $d_{x z}$ & os & 0.080 & -4.30 & 2.55 & 1.16 & 1.13 & & $0.10 \pi$ & 0.90 & 0.66 \\
\hline & $d_{x y}$ & $e a$ & 0.126 & -4.22 & 2.60 & $\ldots$ & $\ldots$ & 25 & $0.43 \pi$ & 0.05 & 0.63 \\
\hline \multirow[t]{4}{*}{$\overline{\mathrm{Fe} /(4,4)}$} & $d_{x z}$ & os & 0.092 & -3.12 & 2.34 & 1.16 & 1.08 & 0.002 & $0.50 \pi$ & & \\
\hline & $d_{x y}$ & $e a$ & 0.082 & -3.94 & 2.72 & $\ldots$ & $\ldots$ & 0.3 & $0.41 \pi$ & 0.09 & 0.93 \\
\hline & $d_{z^{2}}$ & es & 0.060 & -2.63 & 2.23 & $\ldots$ & & & $0.03 \pi$ & 0.03 & 0.50 \\
\hline & $s$ & es & 0.039 & 0.79 & 0.12 & $\ldots$ & & & & & \\
\hline \multirow{3}{*}{$\begin{array}{l}\mathrm{Fe} /(4,4) \\
\text { inside }\end{array}$} & $d_{x z}$ & os & 0.126 & -2.49 & 2.41 & 1.14 & 0.71 & 0.05 & $0.50 \pi$ & & \\
\hline & $d_{x y}$ & $e a$ & 0.396 & -2.01 & 1.86 & $\ldots$ & $\ldots$ & 30 & $0.51 \pi$ & 0.00 & 0.76 \\
\hline & $d_{z^{2}}$ & es & 0.363 & -2.11 & 1.33 & $\ldots$ & & & $0.12 \pi$ & 0.16 & 0.56 \\
\hline \multirow[t]{3}{*}{$\mathrm{Fe} /(8,8)$} & $d_{x z}$ & os & 0.062 & -2.90 & 2.42 & 1.15 & 1.00 & $\sim 10^{-7}$ & $0.50 \pi$ & & \\
\hline & $d_{x y}$ & $e a$ & 0.044 & -2.95 & 2.45 & $\ldots$ & $\ldots$ & $\sim 10^{-8}$ & $0.50 \pi$ & 0.00 & 0.98 \\
\hline & $d_{z^{2}}$ & es & 0.057 & -2.37 & 1.88 & $\ldots$ & & & $0.03 \pi$ & 0.01 & 0.69 \\
\hline \multirow{3}{*}{$\begin{array}{l}\mathrm{Fe} /(8,8) \\
\text { inside }\end{array}$} & $d_{x z}$ & os & 0.081 & -3.14 & 2.56 & 1.14 & 1.18 & $\sim 10^{-5}$ & $0.49 \pi$ & & \\
\hline & $d_{x y}$ & $e a$ & 0.134 & -3.19 & 2.65 & $\ldots$ & $\ldots$ & 0.01 & $0.48 \pi$ & 0.01 & 0.90 \\
\hline & $d_{z^{2}}$ & es & 0.112 & -1.81 & 1.02 & $\ldots$ & & & $0.04 \pi$ & 0.04 & 0.88 \\
\hline \multirow{4}{*}{$\begin{array}{l}(4,4) \text { Long. } \\
\text { Vacancy } \\
(4,4) \text { Transv. } \\
\text { Vacancy }\end{array}$} & $\sigma$ & $e$ & 0.065 & -1.56 & 2.60 & 0 & 0 & $\sim 10^{-3}$ & $0.51 \pi$ & & 1.00 \\
\hline & $\pi$ & $e$ & 0.523 & & & $\ldots$ & $\ldots$ & & & & 0.65 \\
\hline & $\sigma$ & I & 0.124 & -1.43 & 2.53 & 0 & 0 & $\sim 1$ & $0.51 \pi$ & & 0.97 \\
\hline & $\pi$ & I & 0.422 & & & $\ldots$ & $\ldots$ & & & & 0.54 \\
\hline
\end{tabular}

where we can check to what extent the errors implict in the DFT starting point influence the calculation. The addition of a $U=2 \mathrm{eV}$ term within GGA $+U$ has the main result of increasing $\left|\epsilon_{i}\right|$ and $U_{i}$, while $\Gamma_{i}$ does not change appreciably. While this has no effect on the zero-temperature value of the zero-bias conductance, it leads to a strong decrease of $T_{K}$ well below $1 \mathrm{~K}$, an inevitable outcome since $T_{K}$ depends on $U_{i}$ exponentially. The actual choice of the parameter $U$ in GGA $+U$ strongly influences the estimate of $T_{K}$, even though it has little effect on the electronic structure especially above a certain value. That is disappointing since there is no rigorous prescription for choosing the value of $U$. The apparent increase of the magnetic spin splitting and of $U_{i}$ upon increasing the parameter $U$ in GGA $+U$ is in fact more an artifact than a true physical effect. In fact, once $U$ has had its main role of pushing atomic occupancies closer to integer values, the physics becomes independent of $U$, while the GGA $+U$ apparent spin splitting keeps on increasing artificially. This is clearly a point that will require further work. For this reason, we decided to determine $T_{K}$ through the parameters obtained by GGA without $U$ with the understanding that this will probably provide an upper estimate.

\section{B. Co inside a $(4,4)$ tube, hollow site}

The equilibrium configuration of Co inside the $(4,4)$ SWNT mirrors the configuration outside and has the same active orbitals $d_{x z}$ and $d_{x y}$. However, in this case, there is a switch of symmetry relative to the outside case. The $d_{x y}$ orbital, essentially inactive outside, is now much more hybridized with the nanotube, hence it loses charge and comes closer to being half-filled. The charge is transferred partly to the $d_{x z}$, now $\sim 70 \%$ occupied, and partly to the nanotube. As a result, Co inside the nanotube is still a $S=1 / 2$ impurity as it was outside, but the magnetization is shared by both orbitals $d_{x z}$ and $d_{x y}$. The parameters that characterize the orbitals are listed in Tables III and VI.

Running NRG for these two orbitals coupled by a Coulomb repulsion $U_{12}$ as well as a ferromagnetic exchange $J$, we find a 
ground-state configuration where $d_{x y}$ is close to half-filled and $d_{x z}$ is close to fully occupied. This $S=1 / 2$ configuration for Co inside the nanotube still leads to a zero-bias conductance $G=G_{0}$, similar to Co outside, with the major difference that the Kondo temperature is now much larger-and the corresponding anomaly in the spectral function is much broadersince inside the orbital $d_{x y}$ is substantially more hybridized than $d_{x z}$ was outside. The switching between $d_{x z}$ and $d_{x y}$ orbitals is mostly a geometrical effect and produces a much stronger hybridization of $d_{x y}$ with the nanotube. The resulting increased delocalization of the $d_{x y}$ orbital implies that the value of $U_{12}$ obtained from fitting the Hartree-Fock mean field is must be somewhat lower than the estimate based on Eq. (10).

Upon repeating the DFT calculation with GGA $+U(U=$ $2 \mathrm{eV}$ ), however, the orbital $d_{x z}$ became almost completely spin polarized, while orbital $d_{x y}$, being delocalized into the nanotube, is unaffected and remains only modestly spin polarized. This is not unexpected, since at the mean-field level the least hybridized orbital generally becomes strongly magnetic. NRG shows that in this case the orbital $d_{x z}$ goes into the Kondo regime with a low value of $T_{K} \sim 10^{-5} \mathrm{~K}$, while orbital $d_{x y}$ moves below the Fermi energy, is about $70 \%$ filled and yields an appreciable decrease of the zero-bias conductance. Thus, suppression of self interactions by inclusion of a Hubbard repulsion in the GGA calculation does not change the spin of the Co impurity, which remains always $S=1 / 2$, but may cause the orbitals to revert back to the case outside the tube, thereby lowering the Kondo temperature. The persistence of a $S=1 / 2$ state contrasts with the case of Co/graphene, ${ }^{16}$ where GGA yields $S=1 / 2$, but GGA $+U$ favors the experimentally relevant $S=1$ configuration. ${ }^{32}$ The reason is that the $d_{x z}$ and $d_{x y}$ orbitals are degenerate on graphene due to the higher $C_{6 v}$ symmetry as opposed to the $C_{2 v}$ symmetry of the nanotube hollow site. In the $3 d^{9} 4 s^{0}$ configuration given by GGA for Co on graphene, the minority-spin $d_{x z}$ and $d_{x y}$ orbitals lie exactly at the Fermi energy; this is an unstable situation when a Hubbard $U$ is added. It turns out that in this case the minority spin doublet moves above the Fermi energy, and charge neutrality is maintained by partially filling the $4 \mathrm{~s}$ orbital, leading to a $3 d^{8} 4 s^{0.5}$ configuration with spin $S=1$. On the $(4,4)$ nanotube, instead, the crystal field removes the degeneracy of the doublet in such a way that an integer occupation of both orbitals can be achieved already for $U=0$. In conclusion, it is likely that a transition $S=1 \rightarrow S=1 / 2$ occurs in going from $\mathrm{Co} /$ graphene (or large nanotubes) to $\mathrm{Co} /$ small single wall nanotubes. As noted earlier, ${ }^{23}$ in small nanotubes it makes a qualitative difference within GGA whether the impurity is adsorbed inside or outside. For Co outside, the orbital $d_{x z}$ is in the Kondo regime with a small Kondo temperature. For Co inside, the Kondo orbital is $d_{x y}$, whose hybridization is substantially larger because of the curvature, hence leading to a larger Kondo temperature inside as opposed to outside. However, if GGA $+U$ is to be trusted, the Kondo orbital would remain $d_{x z}$ in both cases, leading to similarly small Kondo temperatures inside and outside.

\section{Co outside a $(4,4)$ tube, on-top site}

In this geometry, the electronic configuration of Co in DFT is the same as it was in the hollow configuration, $3 d^{9} 4 s^{0}$, with active orbitals $d_{y z}$ and $d_{x y}$ (see Tables III and VII). However, because of the lower symmetry, the $s$ and $a$ bands are mixed. In this case we need to use a more general expression for the conductance:

$$
\begin{aligned}
G= & {\left[\cos ^{2}\left(\delta_{e s}-\delta_{o s}\right)+\cos ^{2}\left(\delta_{e a}-\delta_{o a}\right)\right] \cos ^{2}\left(\theta_{e}-\theta_{o}\right) } \\
& +\left[\cos ^{2}\left(\delta_{e s}-\delta_{o a}\right)+\cos ^{2}\left(\delta_{e a}-\delta_{o s}\right)\right] \sin ^{2}\left(\theta_{e}-\theta_{o}\right),
\end{aligned}
$$

where $\theta_{e, o}$ are the mixing angles between es-ea and os-oa channels, which can be estimated from DFT calculations. However, in DFT, these angles depend on the spin polarization because spin up and down are widely split and probe different energy regions. This would force us to use a more complicated model than the simple Anderson Hamiltonian (5) with $k$-independent matrix elements $V_{i k a} \equiv V_{i a}$. To simplify the analysis, we decided to drop the $d_{x y}$ orbital, whose effect is presumably small. This is equivalent to assuming that a particular linear combination of $s$ and $a$ bands is coupled to $d_{y z}$, while the orthogonal combination is free and gives unitary conductance. Within this approximation, we find that the results are similar to the hollow configuration as far as conductance $\left(\sim G_{0}\right)$ but indicate a slightly larger Kondo temperature $(\sim 3 \mathrm{~K})$.

\section{Co outside an $(8,8)$ tube, hollow site}

The configuration of Co on the $(8,8)$ SWNT resembles that for the $(4,4)$ SWNT and has the same active orbitals $d_{x z}$ (magnetic) and $d_{x y}$ (almost fully occupied). Therefore the behavior of Co on the $(8,8)$ SWNT is similar to that on the $(4,4)$ SWNT (see Tables III and VIII). Both $U$ and $\Gamma$ are slightly smaller than in the $(4,4)$ tube, implying roughly the same conductance (close to $G_{0}$ ) and a somewhat smaller Kondo temperature (about $0.1 \mathrm{~K}$ when taking into account $J$ and $U_{12}$ ). It follows that the conductance does not depend appreciably on the size of the nanotube, provided it is small.

\section{E. Co inside an $(8,8)$ tube, hollow site}

The configuration of Co inside the $(8,8)$ SWNT is similar to that of Co inside the $(4,4)$ SWNT and has the same active orbitals $d_{x z}$ and $d_{x y}$. However, in this case, the latter orbital is less hybridized with the nanotube due to the reduced curvature. The various parameters that characterize the orbitals are listed in Tables III and IX.

\section{Fe INSIDE AND OUTSIDE NANOTUBES: RESULTS}

After the above exhaustive study of the Co impurity, it is instructive to compare results with a Fe impurity, which highlights some common aspects as well as differences.

\section{A. Fe outside a $(4,4)$ tube, hollow site}

In the adsorbed Fe impurity, the orbitals $d_{x z}$ and $d_{x y}$ are both magnetic. In addition, GGA predicts that $d_{z^{2}}$ is also close to being magnetic and that $4 s$ is partly occupied and polarized, leading to a fractional total magnetic moment $\mu=3.40 \mu_{B}$. Therefore, unlike all previous examples, GGA results are here compatible with a mixture of $3 d^{8} 4 s^{0}(S=1)$ and $3 d^{7} 4 s^{1}(S=2)$. The AIM hence involves a total of four 
TABLE IV. Relevant parameters for the conductance at finite bias; for each case, we show the magnetic orbital, the width of the Kondo resonance $\Gamma_{K}$, and the Fano parameters $q$ (GGA results are shown).

\begin{tabular}{lccccr}
\hline \hline Impurity & Nanotube & Position & Orbital & $\Gamma_{K}(\mathrm{eV})$ & \multicolumn{1}{c}{$\mathrm{q}$} \\
\hline $\mathrm{Co}$ & $(4,4)$ & Out & $d_{x z}$ & $1.5 \times 10^{-4}$ & -0.03 \\
$\mathrm{Co}$ & $(8,8)$ & Out & $d_{x z}$ & $2.5 \times 10^{-5}$ & -0.04 \\
$\mathrm{Co}$ & $(8,8)$ & In & $d_{x y}$ & $6.3 \times 10^{-3}$ & -0.10 \\
$\mathrm{Co}$ & $(4,4)$ & $\mathrm{In}$ & $d_{x y}$ & 0.15 & -0.11 \\
\hline $\mathrm{Fe}$ & $(4,4)$ & Out & $d_{x z}$ & $5 \times 10^{-7}$ & 0.01 \\
& & & $d_{x y}$ & $8 \times 10^{-5}$ & -0.02 \\
$\mathrm{Fe}$ & $(8,8)$ & Out & $d_{x z}$ & $3 \times 10^{-11}$ & 0.06 \\
& & & $d_{x y}$ & $3 \times 10^{-12}$ & -0.02 \\
$\mathrm{Fe}$ & $(8,8)$ & In & $d_{x z}$ & $3 \times 10^{-9}$ & 0.09 \\
& & & $d_{x y}$ & $4 \times 10^{-6}$ & -0.01 \\
$\mathrm{Fe}$ & $(4,4)$ & In & $d_{x z}$ & $1.3 \times 10^{-5}$ & 0.35 \\
& & & $d_{x y}$ & $8 \times 10^{-3}$ & -0.06 \\
\hline \hline
\end{tabular}

orbitals coupled to three channels, two of them ( $4 s$ and $3 d_{z^{2}}$ ) with the same es symmetry.

The effective AIM for Fe is thus much more complicated since four orbitals are involved. In addition to three $d$ orbitals, the $4 s$ orbital is also partly occupied, its spin-up component being exactly at the Fermi energy, so that the choice of genuine magnetic orbitals is not straightforward. Some orbitals become magnetic, i.e., partially filled, only in response to the magnetization of other orbitals. Since we know that spin symmetry must be recovered in the ground state, these orbitals should in the true ground state end up fully empty or fully occupied and hence not Kondo active. Whereas in all the previous examples the distinction between genuine magnetic orbitals and orbitals that magnetize indirectly was clear, in the case of $\mathrm{Fe}(4,4)$ there are uncertainties, especially regarding the orbitals $3 d_{z^{2}}$ and $4 s$. If we just focus on these two, we need to solve an AIM with two nondegenerate orbitals hybridized to a single conduction channel and coupled to each other by a ferromagnetic exchange. Using the parameters extracted from GGA, we ran an NRG calculation for this model and found that in the ground state the $4 s$ orbital is practically empty and the $3 d_{z^{2}}$ fully occupied. Therefore we expect that, in contrast to the GGA starting point, the actual atomic configuration of Fe on the $(4,4)$ SWNT will be $3 d^{8} 4 s^{0}$, which is the same we will find for the $(8,8)$ tube, with two magnetic orbitals, $d_{x z}$ and $d_{x y}$, and hence spin $S=1$. The NRG calculation for these two orbitals, each hybridized to a conduction channel and mutually coupled by Hund's rule ferromagnetic exchange, yields full Kondo screening with each channel acquiring a phase shift close to $\pi / 2$. Small deviations from $\pi / 2$ are caused by imperfect particle-hole symmetry. The final result is that the conductance at low bias and low temperature is pushed down to $G \simeq 0$.

However, we cannot rule out the occurrence in $\mathrm{Fe}$ of an $S=3 / 2$ state with the orbital $3 d_{z^{2}}$ magnetic in addition to orbitals $3 d_{x z}$ and $3 d_{x y}$. That case is beyond our numerical capabilities, requiring three screening channels in the NRG calculation, but would most likely lead to a very low Kondo temperature and a zero-bias conductance of $G \sim G_{0}$. In this case, anisotropy would probably prevail and destroy the Kondo effect. Results are summarized in Tables III and X.

\section{B. Fe outside an $(8,8)$ tube, hollow site}

In this configuration, $\mathrm{Fe}$ is close to $3 d^{8} 4 s^{0}$, thus carrying a magnetic moment $S=1$. As in the $(4,4)$ tube, there are two magnetic orbitals, $d_{x y}$, coupled to the ea channel and $d_{x z}$, coupled to the os channel, and an almost fully occupied orbital, $d_{z^{2}}$, coupled to the es channel. The other orbitals can be safely assumed to be inactive, so the AIM comprises three orbitals, each coupled to a different conduction channel and coupled among themselves by a ferromagnetic exchange $J$ and a Coulomb repulsion $U_{12}$ (see Tables III and XI).

Among the active orbitals, $d_{x z}, d_{z^{2}}$, and $d_{x y}$, the latter couples to the antisymmetric band and pushes the conductance down to zero in that channel. The orbitals $d_{x z}$ and $d_{z^{2}}$ both couple to the symmetric band; the former causes a phase shift of about $\pi / 2$ in the odd channel, the latter a phase shift close to zero in the even channel. It follows that the total conductance is nearly vanishing. Since there are two magnetic orbitals, the system should exhibit two Kondo temperatures. However, it turns out that these are even lower than in the case of $\mathrm{Co}$, and well below $1 \mathrm{~K}$, due to the ferromagnetic Hund exchange $J$ between the two channels in Fe. Such low values of the Kondo temperature mean that other effects, for example spin anisotropy, which has been neglected in our work, will prevail and destroy the Kondo effect. Results are summarized in Table XI.

\section{Fe inside a $(4,4)$ tube, hollow site}

The configuration of Fe inside the $(4,4)$ SWNT is similar to the configuration outside and has the same active orbitals $d_{x z}$ and $d_{x y}$. However, $d_{z^{2}}$ is now predicted to be almost completely filled and the $4 s$ orbital is empty and far from the Fermi energy, giving a total magnetic moment $\mu=1.84 \mu_{B}$.

TABLE V. Case of the $\mathrm{Co} /(4,4)$ system. Relevant DFT quantities (energy of $d$ orbitals $\epsilon_{\sigma}$, in $\mathrm{eV}$, and phase shifts $\delta_{\text {sigma }}$ for each spin direction), parameters extracted from the Hartree-Fock analysis (broadening of the level $\Gamma$, in $\mathrm{eV}$, which is compared to the broadening directly extracted from the PDOS, $\Gamma_{\mathrm{PDOS}}$, and the potential-scattering phase shift $\phi$, in radians), and results from the NRG runs (phase shift $\delta_{\mathrm{NRG}}$, in radians, and conductance $g_{\mathrm{NRG}}$, in units of $G_{0}$, compared to the DFT prediction $g_{\mathrm{DFT}}$ ).

\begin{tabular}{|c|c|c|c|c|c|c|c|c|c|c|}
\hline orbital & $\epsilon_{\uparrow}$ & $\epsilon_{\downarrow}$ & $\delta_{\uparrow}$ & $\delta_{\downarrow}$ & $\Gamma_{\text {PDOS }}$ & $\Gamma$ & $\phi$ & $\delta_{\mathrm{NRG}}$ & $g_{\mathrm{NRG}}$ & $g_{\mathrm{DFT}}$ \\
\hline$d_{x^{2}-y^{2}}(e s)$ & -1.16 & -0.36 & 0.118 & 0.023 & & 0.050 & 0.160 & & & \\
\hline$d_{x z}(o s)$ & -1.56 & 0.47 & 0.133 & 0.358 & 0.080 & 0.087 & 0.187 & -1.41 & $0.03(s)$ & 0.95 \\
\hline$d_{x y}(e a)$ & -1.18 & -0.04 & 0.156 & -0.827 & 0.062 & 0.051 & 0.198 & -0.35 & & \\
\hline$d_{y z}(o a)$ & -2.16 & -1.36 & 0.016 & -0.038 & & 0.197 & 0.107 & & $0.93(a)$ & 0.72 \\
\hline
\end{tabular}


TABLE VI. Same as Table V for Co inside the $(4,4)$ tube.

\begin{tabular}{|c|c|c|c|c|c|c|c|c|c|c|}
\hline orbital & $\epsilon_{\uparrow}$ & $\epsilon_{\downarrow}$ & $\delta_{\uparrow}$ & $\delta_{\downarrow}$ & $\Gamma_{\mathrm{PDOS}}$ & $\Gamma$ & $\phi$ & $\delta_{\mathrm{NRG}}$ & $g_{\mathrm{NRG}}$ & $g_{\text {DFT }}$ \\
\hline$d_{x^{2}-y^{2}}(e s)$ & -1.31 & -0.86 & -0.109 & -0.321 & & 0.392 & -0.210 & & & \\
\hline$d_{x z}(o s)$ & -1.39 & 0.03 & 0.053 & 1.274 & 0.079 & 0.095 & 0.121 & -0.31 & $0.99(s)$ & 0.49 \\
\hline$d_{x y}(e a)$ & -0.71 & -0.12 & -0.309 & -1.245 & 0.407 & 0.380 & 0.210 & -1.29 & & \\
\hline$d_{y z}(o a)$ & -1.69 & -1.19 & 0.081 & 0.073 & & 0.031 & 0.099 & & $0.14(a)$ & 0.46 \\
\hline
\end{tabular}

As a consequence, the configuration is $3 d^{8} 4 s^{0}$ and carries a spin $S=1$, roughly the same as $\mathrm{Fe}$ on an $(8,8)$ tube. Like in the case with Co inside a $(4,4)$ tube, the $d_{x y}$ orbital is strongly hybridized with the nanotube, leading to a high Kondo temperature, on the order of $30 \mathrm{~K}$. However, in this case, GGA $+U$ does not qualitatively affect the result, both orbitals $d_{x z}$ and $d_{x y}$ being always magnetic. The various parameters that characterize the orbitals are listed in Tables III and XII.

\section{Fe inside an $(8,8)$ tube in the hollow position}

The configuration of Fe inside the $(8,8)$ SWNT is similar to the one of Fe outside the $(8,8)$ SWNT and has the same active orbitals $d_{x z}$ and $d_{x y}$. However, the hybridization of the $d_{x y}$ orbital, and hence its Kondo temperature, is now smaller due to the reduced curvature. The various parameters that characterize the orbitals are listed in Tables III and XIII.

Summing up, the Fe impurity is a multichannel Kondo system, as opposed to a single channel for Co, but otherwise similar to Co. The highest predicted Kondo temperatures lie somewhat below those expected for Co, owing to Hund's rule ferromagnetic exchange among the two channels.

\section{PREDICTED KONDO ZERO-BIAS CONDUCTANCE ANOMALIES}

In the previous section, we discussed the Kondo temperature and the zero-bias conductance of Co and Fe impurities. Now we extend the discussion to finite-bias effects, by means of the Keldysh method for nonequilibrium Green's functions. ${ }^{33}$ The conductance for a single band can be expressed in the form of a Fano ${ }^{34}$ resonance

$g_{s, a}(v) \equiv \frac{G_{s, a}(v)}{G_{0}}=\frac{(q+v)^{2}}{\left(q^{2}+1\right)\left(v^{2}+1\right)}, \quad v \equiv \frac{-e V_{B}-\epsilon_{K}}{\Gamma_{K}}$,

where $v$ is the dimensionless bias potential $\left(V_{B}, \Gamma_{K}\right.$, and $\epsilon_{K}$ are, respectively, the bias potential, the width of the resonance, proportional to the Kondo temperature, and the energy of the Kondo peak, in eV); $q$ is the Fano parameter, which describes the shape of the ZBA: $q=0$ means an anti-Lorentzian shape, $q= \pm \infty$ a Lorentzian one, and $q= \pm 1$ gives rise to the most asymmetric line shapes.

The above formula Eq. (11) holds for a single band. If we assume no coupling between the two bands (that is, $J=$ $U_{12}=0$ ), we can get the total conductance by simply adding the results from each band:

$$
g_{\text {tot }}(v)=g_{s}(v)+g_{a}(v) .
$$

This is no longer strictly true if the bands are coupled to each other. However, since the treatment becomes quite involved in that case, we will simply assume that Eq. (12) still holds approximately. Results are shown in Table IV.

\section{KONDO EFFECT OF VACANCIES IN CARBON NANOTUBES}

Single-atom vacancies in a nanotube represent a simpler and more intrinsic magnetic impurity than the adsorbed transition metal atom described in the previous sections. We apply the same method described for Co and Fe impurities to a singleatom vacancy in a $(4,4)$ nanotube. However, due to the lower symmetry of this situation, calculations can only be pursued to a more modest degree of accuracy.

When removing a carbon atom from a graphene sheet, carbon nanotube, or nanoribbon, a magnetic moment arises ${ }^{35-38}$ due to the breaking of three $\sigma\left(2 s p^{2}\right.$ hybrid $)$ bonds and one $\pi\left(2 p_{z}\right)$ bond. The magnetic moment has been found by DFT to be close to $1 \mu_{B},{ }^{35,36}$ hinting at $S=1 / 2$, even though in some situations magnetism seems to disappear. ${ }^{39}$ The magnetic moment, being embedded in a metal (armchair nanotube) or in a semimetal (graphene), should give rise to a Kondo effect. The case of graphene has been investigated both theoretically ${ }^{40-42}$ and experimentally, ${ }^{43}$ but the vacancy Kondo effect has not been addressed so far in nanotubes.

Out of the three dangling $\sigma$ bonds created by the vacancy, two are mutually saturated when the two respective $\mathrm{C}$ atoms come together forming a weak Jahn-Teller type bond. In general, three different pairs can form, corresponding to three different static distortions of the carbons lying next to the

TABLE VII. Same as Table V for Co in the on-top position on the $(4,4)$ tube. Due to the lower symmetry of this case, it is not possible to assign unequivocally an orbital to each channel; we just show the orbital with the highest weight coupled to each channel.

\begin{tabular}{|c|c|c|c|c|c|c|c|c|c|c|}
\hline orbital & $\epsilon_{\uparrow}$ & $\epsilon_{\downarrow}$ & $\delta_{\uparrow}$ & $\delta_{\downarrow}$ & $\Gamma_{\mathrm{PDOS}}$ & $\Gamma$ & $\phi$ & $\delta_{\mathrm{NRG}}$ & $g_{\text {NRG }}$ & $g_{\text {DFT }}$ \\
\hline$d_{x^{2}-y^{2}}(e)$ & -1.28 & -0.60 & 0.161 & 0.060 & & 0.116 & 0.248 & & & \\
\hline$d_{x z}(o)$ & -1.42 & -0.84 & 0.028 & -0.063 & & 0.187 & 0.159 & & & \\
\hline$d_{x y}(e)$ & -1.15 & -0.07 & 0.021 & -0.655 & & 0.059 & 0.072 & & 1 & 0.79 \\
\hline$d_{y z}(o)$ & -1.96 & 0.41 & 0.077 & 0.294 & 0.083 & 0.076 & 0.116 & -1.48 & 0.02 & 0.99 \\
\hline
\end{tabular}


TABLE VIII. Same as Table V for the $\mathrm{Co} /(8,8)$ system.

\begin{tabular}{|c|c|c|c|c|c|c|c|c|c|c|}
\hline orbital & $\epsilon_{\uparrow}$ & $\epsilon_{\downarrow}$ & $\delta_{\uparrow}$ & $\delta_{\downarrow}$ & $\Gamma_{\mathrm{PDOS}}$ & $\Gamma$ & $\phi$ & $\delta_{\mathrm{NRG}}$ & $g_{\mathrm{NRG}}$ & $g_{\text {DFT }}$ \\
\hline$d_{x^{2}-y^{2}}(e s)$ & -1.52 & -0.64 & 0.091 & 0.046 & & 0.051 & 0.125 & & & \\
\hline$d_{x z}(o s)$ & -1.68 & 0.26 & 0.129 & 0.365 & 0.057 & 0.058 & 0.162 & -1.41 & $0.04(s)$ & 0.95 \\
\hline$d_{x y}(e a)$ & -1.36 & 0.01 & 0.121 & 1.368 & 0.039 & 0.038 & 0.149 & -0.28 & & \\
\hline$d_{y z}(o a)$ & -2.00 & -0.94 & 0.076 & 0.036 & & 0.071 & 0.111 & & $1.00(a)$ & 0.53 \\
\hline
\end{tabular}

vacancy. In graphene, these three configurations are equivalent, since they can be transformed into one another by rotating the whole system by $\pm 2 \pi / 3$ given the local $C_{3 v}$ symmetry. In an armchair nanotube, where only a symmetry $C_{S}$ is preserved, two out of three configurations are still equivalent, and we will call them "transverse" (T). The third configuration is inequivalent and we will call it "longitudinal" (L) (see Fig. 1). In our $(4,4)$ SWNT, a DFT calculation shows that the transverse configuration is energetically favorable over the longitudinal one by about $0.8 \mathrm{eV}$.

Nonetheless, we will consider both $\mathrm{T}$ and $\mathrm{L}$ cases to illustrate the differences that arise. In all cases, the JT relaxation saturates two $\sigma$ dangling bonds. Two remaining broken bonds are left unsaturated-one $\sigma$ on the $\mathrm{C}$ atom (called $C_{1}$ ), which is left unpaired, and one $\pi$. In both $\mathrm{T}$ and L configurations, the DFT calculated magnetic moment is close to $1 \mu_{B}$ and mainly carried by the $\sigma$ orbital localized on the lone atom $C_{1}$. In addition, a $\pi$ symmetry state appears just below the Fermi energy (see Fig. 2). The corresponding wave function is delocalized around the defect, indicating that unlike the sigma broken bond, which is localized, the broken $\pi$ bond undergoes strong delocalization within the $\pi$ nanotube conduction band. The hybridization of the broken $\pi$ bond with the nanotube bands appears strong enough to inhibit the spontaneous formation of a full magnetic moment. The corresponding electronic states exhibit a small spin splitting and some magnetization but only as a result of intra-atomic exchange with the strongly magnetized $\sigma$ broken bond. We find that in the transverse configuration, the $\pi$ vacancy state is antiferromagnetically coupled to the $\sigma$ orbital spin, leading to a total magnetic moment smaller than $1 \mu_{B}$. In the longitudinal configuration, instead, the coupling is very weakly ferromagnetic, yielding a total magnetic moment which is slightly larger than $1 \mu_{B}$. This difference can be traced to the fact that the correlations are ferromagnetic within a sublattice and antiferromagnetic between the two sublattices. The exchange coupling is ferromagnetic for two orbitals localized on the same atom due to Hund's rule, but antiferromagnetic for two orbitals on neighboring atoms, as in the Hubbard model. The $\pi$ orbital is delocalized around the defect, and its exchange coupling with the $\sigma$ state will be ferromagnetic or antiferromagnetic according to its weight on the various $\mathrm{C}$ atoms, a property that is evidently controlled by geometry.

In Fig. 4, we show the spin-polarized transmission for the two types of vacancies. One conduction channel is always decoupled from the $\sigma$ and $\pi$ impurity orbitals, giving a contribution $\sim G_{0}$ to the total conductance. Both up- and down-spin $\sigma$ orbitals being far from the Fermi energy, the DFT conductance at zero bias is dictated by the $\pi$ orbital. In the longitudinal configuration, both up- and down-spin $\pi$ orbitals are about $0.7 \mathrm{eV}$ below the Fermi energy, contributing $\sim 0.65 G_{0}$ to the total zero-bias conductance $G=1.65 G_{0}$. In the transverse configuration, the up-spin orbital is about $0.2 \mathrm{eV}$ below Fermi, leading to $G_{\uparrow}=0.2 G_{0} / 2$, while the down-spin orbital is $1.1 \mathrm{eV}$ below Fermi, leading to $G_{\downarrow}=0.9 G_{0} / 2$, which together with the decoupled channel gives a total conductance of $G=1.5 G_{0}$. For simplicity, we keep just the $\sigma$ orbital in building the AIM, which leads to a one-channel Hamiltonian whose parameters are shown in Table III.

The small $T_{K}$ that we find in both cases contrasts with the case of graphene, where the experimentally determined $T_{K}$ is between 30 and $90 \mathrm{~K} .{ }^{43}$ We tentatively attribute this difference to the large curvature of the nanotube, which should substantially modify the hybridization of the $\sigma$ orbital with conduction channels.

\section{DISCUSSION AND CONCLUSIONS}

We have shown in a detailed case study how one can combine $a b$ initio electronic structure calculations with numerical renormalization group to get quantitative estimates of the Kondo temperature and zero-bias anomaly in the transport across atomically, structurally and electronically controlled nanowires. The specific examples we have chosen to apply this strategy to are $\mathrm{Co}$ and Fe magnetic impurities adsorbed on single-wall carbon nanotubes and a carbon vacancy in a pristine nanotube. While there are no experimental data for these systems, their extreme simplicity and reproducibility recommend them as ideal test cases for future study. Even in the absence of experimental data, the effect of various approximations and DFT errors can be tested here rather instructively.

Our main results can be summarized very shortly. A Co atom (or a $\mathrm{C}$ vacancy) behaves effectively as a $S=1 / 2$

TABLE IX. Same as Table V for Co inside the $(8,8)$ tube.

\begin{tabular}{|c|c|c|c|c|c|c|c|c|c|c|}
\hline orbital & $\epsilon_{\uparrow}$ & $\epsilon_{\downarrow}$ & $\delta_{\uparrow}$ & $\delta_{\downarrow}$ & $\Gamma_{\mathrm{PDOS}}$ & $\Gamma$ & $\phi$ & $\delta_{\mathrm{NRG}}$ & $g_{\mathrm{NRG}}$ & $g_{\text {DFT }}$ \\
\hline$d_{x^{2}-y^{2}}(e s)$ & -1.68 & -1.05 & 0.026 & -0.037 & & 0.178 & 0.131 & & & \\
\hline$d_{x z}(o s)$ & -1.63 & 0.07 & 0.068 & 0.921 & 0.070 & 0.080 & 0.117 & -0.31 & $0.90(s)$ & 0.66 \\
\hline$d_{x y}(e a)$ & -1.37 & 0.08 & 0.049 & 1.063 & 0.099 & 0.126 & 0.140 & -1.35 & & \\
\hline$d_{y z}(o a)$ & -1.69 & -1.19 & 0.081 & 0.030 & & 0.010 & 0.137 & & $0.05(a)$ & 0.63 \\
\hline
\end{tabular}


TABLE X. Same as Table V for the Fe/(4,4) system.

\begin{tabular}{|c|c|c|c|c|c|c|c|c|c|c|}
\hline orbital & $\epsilon_{\uparrow}$ & $\epsilon_{\downarrow}$ & $\delta_{\uparrow}$ & $\delta_{\downarrow}$ & $\Gamma_{\text {PDOS }}$ & $\Gamma$ & $\phi$ & $\delta_{\mathrm{NRG}}$ & $g_{\mathrm{NRG}}$ & $g_{\text {DFT }}$ \\
\hline$d_{z^{2}}(e s)$ & -3.08 & 0.23 & -1.443 & 0.363 & 0.066 & 0.060 & 0.149 & -0.09 & & \\
\hline $\mathrm{s}(e s)$ & 0.01 & 1.64 & & & 0.039 & (0.039) & & & & \\
\hline$d_{x z}(o s)$ & -2.10 & 1.10 & 0.188 & 0.308 & & 0.092 & 0.230 & -1.57 & $0.03(s)$ & 0.50 \\
\hline$d_{x y}(e a)$ & -2.90 & 0.70 & 0.197 & 0.333 & 0.065 & 0.082 & 0.224 & -1.29 & & \\
\hline$d_{y z}(o a)$ & -2.88 & -1.24 & 0.100 & -0.039 & & 0.301 & 0.202 & & $0.09(a)$ & 0.93 \\
\hline
\end{tabular}

impurity and reduces the zero-bias conductance from the ideal value of $G=2 G_{0}=4 e^{2} / h$ down to $G=G_{0}$. On the contrary, $\mathrm{Fe}$ is found to be $S=1$ and should be able to completely suppress the conductance, $G=0$. This reduction in the conductance takes place over a range of temperature/bias determined by the typical scale $T_{K}$ of Kondo screening. We generally estimate $T_{K}$ to be small, a fraction of a Kelvin, and hence hard to detect. The only exception is Co or Fe inside the narrowest $(4,4)$ tube, where the curvature leads to a substantial enhancement of the hybridization between the magnetic orbital $d_{x y}$ and the nanotube, pushing the Kondo temperature fairly high. Even this result has some level of uncertainty, since we do not yet know if the self-interaction error in DFT is excessive or not. In all other cases, the tiny values of $T_{K}$ make our results difficult to verify experimentally, which is a bit disappointing. There is, however, a positive implication, namely that, according to these results, the increase in the resistivity observed in nanotubes below $100 \mathrm{~K}^{44-46}$ as well as the associated peak in the thermopower ${ }^{44,45}$ could indeed be caused by magnetic impurities trapped inside the tube. A remarkable magnetic impurity is the single carbon atom vacancy. Although its Jahn Teller distorted structure and $S=1 / 2$ should, in principle, resemble that of a vacancy in graphene, the predicted Kondo temperature is substantially smaller in the nanotube, most likely owing to the lower hybridization caused by curvature.

From a general methodological perspective, our study highlights several interesting elements and difficulties in the $a b$ initio modeling of magnetic impurities in nanoscale conductors. The method we have presented combines the advantages of DFT and NRG in a simple and easily manageable way; the former allows us to identify the magnetic orbitals and their electronic properties, while the latter correctly incorporates the quantum fluctuations that restore spin symmetry. In this respect, it could be quite effective in many other cases, e.g. magnetic molecules. The most important difficulty is that the Anderson model parameters obtained from spin-polarized GGA, where the Kohn-Sham energy levels are generally affected by unknown self-interaction errors, are not always reliable and often need to be corrected, for example by means of GGA $+U$, as repeatedly stressed above. The other important aspect that is worth emphasizing is the distinction between what we might call "driving" and "driven" magnetic orbitals. Since spin-polarized GGA breaks spin symmetry, there are orbitals that become partially polarized only in response to the full magnetization of other orbitals to which they are coupled by Coulomb (Hund's rule) exchange. The net effect is that within GGA one often finds a fractional magnetic moment, which cannot be straightforwardly associated with a definite-spin Kondo impurity. On the other hand, since the actual ground state must be spin-rotationally invariant, it is likely that orbitals that appear weakly polarized in GGA are in reality either empty or fully occupied, and hence nonmagnetic. Only a better calculation that takes quantum fluctuations properly into account can clarify this issue, which is exactly what NRG is suited for. In conclusion, the combined DFT + NRG approach that we have exemplified is able to provide a satisfactory, if approximate, description of the actual low-temperature and low-bias behavior of a magnetic nanocontact. Nevertheless, we should stress that several quantitative aspects, like the precise width and line shape of the Kondo anomaly, remain uncertain mainly because the actual magnitude of the Hubbard $U$ in the Anderson model depends on the way GGA is implemented and small changes of $U$ may lead to appreciable changes of $T_{K}$.

\section{ACKNOWLEDGMENTS}

This work was largely supported by PRIN/COFIN 2010LLKJBX. It also benefited by the environment created by EU-Japan Project LEMSUPER, Sinergia Contract CRSII2 $136287 / 1$, and ERC Advanced Grant 320796 MODPHYSFRICT.

\section{APPENDIX A: NUMERICAL RENORMALIZATION GROUP CALCULATIONS}

In this appendix, we show how the impurity model of Eq. (5) is solved by means of numerical renormalization group calculations. NRG is a numerical technique originally developed by K. G. Wilson ${ }^{11}$ to solve Anderson and Kondo impurity models. It is by now a well established impurity solver, whose technical details can be found in many review papers, see, e.g., Ref. 47. The NRG method imposes a

TABLE XI. Same as Table V for the Fe/(8,8) system.

\begin{tabular}{|c|c|c|c|c|c|c|c|c|c|c|}
\hline orbital & $\epsilon_{\uparrow}$ & $\epsilon_{\downarrow}$ & $\delta_{\uparrow}$ & $\delta_{\downarrow}$ & $\Gamma_{\mathrm{PDOS}}$ & $\Gamma$ & $\phi$ & $\delta_{\mathrm{NRG}}$ & $g_{\mathrm{NRG}}$ & $g_{\text {DFT }}$ \\
\hline$d_{z^{2}}(e s)$ & -1.96 & -0.06 & 0.112 & -0.681 & 0.059 & 0.057 & 0.141 & -0.09 & & \\
\hline$d_{x z}(o s)$ & -1.60 & 1.14 & 0.135 & 0.225 & & 0.062 & 0.172 & 1.57 & $0.01(s)$ & 0.69 \\
\hline$d_{x y}(e a)$ & -1.72 & 1.12 & 0.137 & 0.200 & & 0.044 & 0.162 & 1.57 & & \\
\hline$d_{y z}(o a)$ & -2.08 & -0.61 & 0.092 & -0.027 & & 0.103 & 0.141 & & $0.00(a)$ & 0.98 \\
\hline
\end{tabular}


TABLE XII. Same as Table V for Fe inside the $(4,4)$ tube.

\begin{tabular}{|c|c|c|c|c|c|c|c|c|c|c|}
\hline orbital & $\epsilon_{\uparrow}$ & $\epsilon_{\downarrow}$ & $\delta_{\uparrow}$ & $\delta_{\downarrow}$ & $\Gamma_{\mathrm{PDOS}}$ & $\Gamma$ & $\phi$ & $\delta_{\mathrm{NRG}}$ & $g_{\mathrm{NRG}}$ & $g_{\text {DFT }}$ \\
\hline$d_{z^{2}}(e s)$ & -1.33 & -0.27 & -0.197 & -0.905 & & 0.363 & 0.073 & -0.38 & & \\
\hline$d_{x z}(o s)$ & -1.35 & 1.06 & 0.007 & 0.215 & & 0.126 & 0.100 & -1.57 & $0.16(s)$ & 0.56 \\
\hline$d_{x y}(e a)$ & -0.84 & 0.90 & -0.241 & 0.588 & 0.442 & 0.396 & 0.222 & 1.54 & & \\
\hline$d_{y z}(o a)$ & -1.69 & -0.47 & 0.083 & -0.115 & & 0.129 & 0.158 & & $0.00(a)$ & 0.76 \\
\hline
\end{tabular}

logarithmic discretization of the conduction band controlled by a discretization parameter $\Lambda$. After a sequence of transformations, the discretized model is mapped onto a semi-infinite chain whose first site is the impurity spin. The Hamiltonian of the chain is diagonalized iteratively starting from the impurity site and successively adding degrees of freedom to the chain. The key parameters that control the accuracy of the calculations are (1) the discretization parameter $\Lambda$, which should be as close as possible to one; and (2) the number of states that are kept at each iteration. In our computations, we used $\Lambda=1.8$ and kept about 1500 states per iteration for runs with a single conduction channel, and $\Lambda=2.5$ and about 3500 states for runs with two conduction channels. The main purpose of the NRG calculations is to determine the low-temperature conductance across the impurity and the energy scale $k_{B} T_{K}$, where $T_{K}$ is the Kondo temperature, that controls the asymptotic low-temperature regime.

The zero-temperature and zero-bias conductance $g \equiv$ $G / G_{0}$ is fully determined by the phase shifts according to the formula

$$
g=\cos ^{2}\left(\delta_{e s}-\delta_{o s}\right)+\cos ^{2}\left(\delta_{e a}-\delta_{o a}\right) \equiv g_{s}+g_{a},
$$

where we assume $\delta_{i j}=\phi_{i j}+\delta_{i j}^{\mathrm{NRG}}(i=e / o, j=s / a)$. Here, the $\phi_{i j}$ are obtained by DFT and $\delta_{i j}^{\mathrm{NRG}}$ are extracted by NRG neglecting the scalar potential $t_{i, k k^{\prime}}$. This assumption is justified since $\phi_{i j}$ are quite small.

In the clean tube, $G=2 G_{0}$ because there are two conduction bands crossing the chemical potential. In the presence of the impurity, the phase shift acquired by each channel $l$ can be estimated by NRG through

$$
\delta_{l}^{\mathrm{NRG}}=\pi \frac{E_{l 1}}{E_{l 2}-E_{l 1}}, \quad l=e s, e a, o s, o a,
$$

where $E_{l 1}$ and $E_{l 2}$ are the first two eigenvalues in the subspace with quantum numbers that correspond to adding one more electron to channel $l$. As mentioned, no NRG calculation is required the channel is decoupled from the impurity orbitals, the phase shift being merely due to potential scattering, $\delta_{l}=$ $\phi_{l} \simeq 0$.

An alternative but equivalent way to estimate $\delta_{l}^{\mathrm{NRG}}$ is to use the real part of the self-energy at zero frequency, $\Re \Sigma(0)$, extracted from the spectral function, since

$$
\delta_{l}^{\mathrm{NRG}}=\arctan \frac{\Gamma_{l}^{*}}{\epsilon_{l}^{*}+\mathfrak{R} \Sigma_{l}(0)} .
$$

These two ways of estimating the phase shifts lead to similar results. Specifically, we always find $\delta_{l} \simeq \pi / 2$ for Kondo channels (channels coupled to a magnetic impurity state), and $\delta_{l} \simeq 0$ for channels coupled only to almost filled or empty orbitals.

We extracted the Kondo temperatures of the magnetic channels from the Matsubara self-energy

$$
\Sigma_{l}(i \epsilon)=G_{0 l}^{-1}(i \epsilon)-G_{l}^{-1}(i \epsilon),
$$

where

$$
G_{0 l}(i \epsilon)=\frac{1}{i \epsilon-\epsilon_{l}+i \Gamma_{l}}
$$

is the noninteracting Green's function (describing the impurity with $U_{l}$ and $J$ set to zero) and

$$
G_{l}(i \epsilon)=\frac{1}{i \epsilon-\epsilon_{l}-\Sigma_{l}(i \epsilon)+i \Gamma_{l}}=\frac{1}{Z_{\text {part }}} \sum_{n} \frac{\left|\left\langle G S\left|d_{l}\right| n\right\rangle\right|^{2}}{i \epsilon-\epsilon_{n}}
$$

is the full Green's function $\left(Z_{\text {part }}\right.$ is the partition function, $G S$ the ground state, and $d_{l}$ annihilates an electron in the impurity orbital with symmetry $l$ ). Matrix elements $\left\langle n\left|d_{l}\right| m\right\rangle$ were collected during the NRG runs according to the patching technique. ${ }^{48}$ This approach introduces small deviations with respect to more refined techniques such as those described in 49 and 50, but, due to our large uncertainties in the determination of parameters, it does not affect substantially our final results.

The Kondo temperature is given by

$$
T_{K}^{l}=\frac{\pi w Z_{l} \Gamma_{l}}{4 k_{B}},
$$

\begin{tabular}{|c|c|c|c|c|c|c|c|c|c|c|}
\hline orbital & $\epsilon_{\uparrow}$ & $\epsilon_{\downarrow}$ & $\delta_{\uparrow}$ & $\delta_{\downarrow}$ & $\Gamma_{\mathrm{PDOS}}$ & $\Gamma$ & $\phi$ & $\delta_{\mathrm{NRG}}$ & $g_{\mathrm{NRG}}$ & $g_{\text {DFT }}$ \\
\hline$d_{z^{2}}(e s)$ & -1.62 & -0.32 & 0.008 & -0.318 & & 0.112 & 0.086 & -0.13 & & \\
\hline$d_{x z}(o s)$ & -1.59 & 1.13 & 0.073 & 0.193 & & 0.081 & 0.124 & -1.54 & $0.04(\mathrm{~s})$ & 0.88 \\
\hline$d_{x y}(e a)$ & -1.56 & 1.12 & 0.039 & 0.240 & & 0.134 & 0.125 & -1.51 & & \\
\hline$d_{y z}(o a)$ & -1.70 & -0.27 & 0.059 & -0.214 & & 0.009 & 0.111 & & $0.01(a)$ & 0.90 \\
\hline
\end{tabular}

where $w=0.4128$ is the Wilson coefficient and $Z_{l}$ is the quasiparticle residue

$$
Z_{l}^{-1}=1-\frac{\partial \Sigma_{l}(i \epsilon)}{\partial(i \epsilon)} .
$$

TABLE XIII. Same as Table V for Fe inside the $(8,8)$ tube. 
The Kondo temperature is related to the width of the Kondo peak $\Gamma_{K}^{l}=Z_{l} \Gamma_{l}$ in the PDOS through

$$
k_{B} T_{K}^{l}=\frac{w \pi}{4} \Gamma_{K}^{l}=0.342 \Gamma_{K}^{l} .
$$

\section{APPENDIX B: FURTHER NUMERICAL DATA}

In Tables V to XIII, we present some further DFT data and related extracted quantities, for different cases.
${ }^{1}$ N. Agrait, A. L. Yeyati, and J. M. van Ruitenbeek, Phys. Rep. 377, 81 (2003).

${ }^{2}$ N. Néel, J. Kröger, L. Limot, K. Palotas, W. A. Hofer, and R. Berndt, Phys. Rev. Lett. 98, 016801 (2007).

${ }^{3}$ A.-P. Jauho, N. S. Wingreen, and Y. Meir, Phys. Rev. B 50, 5528 (1994).

${ }^{4}$ B. Larade, J. Taylor, H. Mehrez, and H. Guo, Phys. Rev. B 64, 075420 (2001).

${ }^{5}$ M. Tsukada, K. Tagami, K. Hirose, and N. Kobayashi, J. Phys. Soc. Jpn. 74, 1079 (2005).

${ }^{6}$ J. A. Appelbaum, Phys. Rev. 154, 633 (1967).

${ }^{7}$ P. W. Anderson, Phys. Rev. Lett. 17, 95 (1966).

${ }^{8}$ H. M. Gupta and U. N. Upadhyaya, Phys. Rev. B 4, 2765 (1971).

${ }^{9}$ D. Goldhaber-Gordon, H. Shtrikman, D. Mahalu, D. AbushMagder, U. Meirav, and M. A. Kastner, Nature (London) 391, 156 (1998).

${ }^{10}$ J. Kondo, Prog. Theor. Phys. 32, 37 (1964).

${ }^{11}$ K. G. Wilson, Rev. Mod. Phys. 47, 773 (1975).

${ }^{12}$ K. S. Thygesen and A. Rubio, Phys. Rev. B 77, 115333 (2008).

${ }^{13}$ D. Jacob, K. Haule, and G. Kotliar, Phys. Rev. Lett. 103, 016803 (2009).

${ }^{14}$ P. W. Anderson, Phys. Rev. 124, 41 (1961).

${ }^{15}$ A. Hewson, The Kondo Problem to Heavy Fermions (Cambridge University Press, Cambridge, 1993).

${ }^{16}$ T. O. Wehling, A. V. Balatsky, M. I. Katsnelson, A. I. Lichtenstein, and A. Rosch, Phys. Rev. B 81, 115427 (2010).

${ }^{17}$ T. A. Costi, L. Bergqvist, A. Weichselbaum, J. von Delft, T. Micklitz, A. Rosch, P. Mavropoulos, P. H. Dederichs, F. Mallet, L. Saminadayar, and C. Bäuerle, Phys. Rev. Lett. 102, 056802 (2009).

${ }^{18}$ R. M. Potok, I. G. Rau, H. Shtrikman, Y. Oreg, and D. GoldhaberGordon, Nature (London) 446, 167 (2007).

${ }^{19}$ N. Roch, S. Florens, T. A. Costi, W. Wernsdorfer, and F. Balestro, Phys. Rev. Lett. 103, 197202 (2009).

${ }^{20}$ J. J. Parks, A. R. Champagne, T. A. Costi, W. W. Shum, A. N. Pasupathy, E. Neuscamman, S. Flores-Torres, P. S. Cornaglia, A. A. Aligia, C. A. Balseiro, G. K.-L. Chan, H. D. Abruña, and D. C. Ralph, Science 328, 1370 (2010).

${ }^{21}$ A. Greuling, M. Rohlfing, R. Temirov, F. S. Tautz, and F. B. Anders, Phys. Rev. B 84, 125413 (2011).

${ }^{22}$ P. Lucignano, R. Mazzarello, A. Smogunov, M. Fabrizio, and E. Tosatti, Nat. Mater. 8, 563 (2009).

${ }^{23}$ P. P. Baruselli, A. Smogunov, M. Fabrizio, and E. Tosatti, Phys. Rev. Lett. 108, 206807 (2012).

${ }^{24}$ P. P. Baruselli, A. Smogunov, M. Fabrizio, and E. Tosatti, Physica E 44, 1040 (2012).
${ }^{25}$ Y. Yagi, T. M. Briere, M. H. F. Sluiter, V. Kumar, A. A. Farajian, and Y. Kawazoe, Phys. Rev. B 69, 075414 (2004).

${ }^{26}$ P. Giannozzi et al., J. Phys.: Condens. Matter 21, 395502 (2009).

${ }^{27}$ J. P. Perdew, K. Burke, and M. Ernzerhof, Phys. Rev. Lett. 77, 3865 (1996).

${ }^{28}$ A. Smogunov, A. Dal Corso, and E. Tosatti, Phys. Rev. B 70, 045417 (2004).

${ }^{29}$ P. W. Anderson, G. Yuval, and D. R. Hamann, Phys. Rev. B 1, 4464 (1970).

${ }^{30}$ D. R. Hamann, Phys. Rev. B 2, 1373 (1970).

${ }^{31}$ A. I. Liechtenstein, V. I. Anisimov, and J. Zaanen, Phys. Rev. B 52, R5467 (1995).

${ }^{32}$ Q. Dubout, Ph.D. thesis, EPFL Lausanne, 2013.

${ }^{33}$ Y. Meir and N. S. Wingreen, Phys. Rev. Lett. 68, 2512 (1992).

${ }^{34}$ U. Fano, Phys. Rev. 124, 1866 (1961).

${ }^{35}$ P. O. Lehtinen, A. S. Foster, Y. Ma, A. V. Krasheninnikov, and R. M. Nieminen, Phys. Rev. Lett. 93, 187202 (2004).

${ }^{36}$ O. V. Yazyev and L. Helm, Phys. Rev. B 75, 125408 (2007).

${ }^{37}$ J. J. Palacios, J. Fernández-Rossier, and L. Brey, Phys. Rev. B 77, 195428 (2008)

${ }^{38}$ O. V. Yazyev, Phys. Rev. Lett. 101, 037203 (2008).

${ }^{39}$ Y. Ma, P. O. Lehtinen, A. S. Foster, and R. M. Nieminen, New J. Phys. 6, 68 (2004).

${ }^{40}$ K. Sengupta and G. Baskaran, Phys. Rev. B 77, 045417 (2008).

${ }^{41}$ P. S. Cornaglia, G. Usaj, and C. A. Balseiro, Phys. Rev. Lett. 102, 046801 (2009).

${ }^{42}$ M. Vojta, L. Fritz, and R. Bulla, Europhys. Lett. 90, 27006 (2010).

${ }^{43}$ J.-H. Chen, L. Li, W. G. Cullen, E. D. Williams, and M. S. Fuhrer, Nat. Phys. 7, 535 (2011).

${ }^{44}$ J. Hone, I. Ellwood, M. Muno, A. Mizel, M. L. Cohen, A. Zettl, A. G. Rinzler, and R. E. Smalley, Phys. Rev. Lett. 80, 1042 (1998).

${ }^{45}$ L. Grigorian, G. U. Sumanasekera, A. L. Loper, S. L. Fang, J. L. Allen, and P. C. Eklund, Phys. Rev. B 60, R11309 (1999).

${ }^{46}$ J. E. Fischer, H. Dai, A. Thess, R. Lee, N. M. Hanjani, D. L. Dehaas, and R. E. Smalley, Phys. Rev. B 55, R4921 (1997).

${ }^{47}$ R. Bulla, T. A. Costi, and T. Pruschke, Rev. Mod. Phys. 80, 395 (2008).

${ }^{48}$ R. Bulla, T. A. Costi, and D. Vollhardt, Phys. Rev. B 64, 045103 (2001).

${ }^{49}$ R. Peters, T. Pruschke, and F. B. Anders, Phys. Rev. B 74, 245114 (2006).

${ }^{50}$ A. Weichselbaum and J. von Delft, Phys. Rev. Lett. 99, 076402 (2007). 